\title{
Nonlinear superharmonic resonance analysis of a nonlocal beam on a fractional visco-Pasternak foundation
}

\author{
Nikola Nešić ${ }^{1}, \underline{\text { Milan Cajić }}^{2}$, Danilo Karličić́c, ${ }^{2,3}$ Goran Janevski ${ }^{4}$ \\ ${ }^{1}$ University of Priština, Faculty of Technical Sciences, 38220 Kosovska Mitrovica, Kneza Milosa 7, Serbia, \\ nikola.nesic@pr.ac.rs \\ ${ }^{2}$ Mathematical Institute of the Serbian Academy of Sciences and Arts, Belgrade, Serbia, \\ mcajic@mi.sanu.ac.rs \\ ${ }^{3}$ College of Engineering, Swansea University, Fabian Way, Crymlyn Burrows, Swansea SA1 8EN, UK \\ danilo.karlicic@swansea.ac.uk, danilok@mi.sanu.ac.rs \\ ${ }^{4}$ University of Niš, Faculty of Mechanical Engineering, A. Medvedeva 14, 18000 Niš, Serbia, \\ gocky.jane@gmail.com
}

\begin{abstract}
This paper investigates the dynamic behaviour of a geometrically nonlinear nanobeam resting on the fractional visco-Pasternak foundation and subjected to dynamic axial and transverse loads. The fractional-order governing equation of the system is derived and then discretized by using the single-mode Galerkin discretization. Corresponding forced Mathieu-Duffing equation is solved by using the perturbation multiple time scales method for the weak nonlinearity and by the semi-numerical incremental harmonic balance method for the strongly nonlinear case. A comparison of the results from two methods is performed in the validation study for the weakly nonlinear case and a fine agreement is achieved. A parametric study is performed and the advantages and deficiencies of each method are discussed for order two and three superharmonic resonance conditions. The results demonstrate a significant influence of the fractionalorder damping of the visco-Pasternak foundation as well as the nonlocal parameter and external excitation load on the frequency response of the system. The proposed methodology can be used in pre-design procedures of novel energy harvesting and sensor devices at small scales exhibiting nonlinear dynamic behaviour.
\end{abstract}

Keywords: nanobeams, nonlocal elasticity, fractional damping, nonlinear vibration, multiple scales method, incremental harmonic balance

\section{Introduction}

The nonlinear dynamic behaviour of beam structures was the subject of investigation within the scientific community for many years $[1,2]$. The approaches to study this problem evolve over the years and different analytical perturbation methods $[3,4]$ or numerical methods [5] were utilized while standard beam models were extended by the inclusion of different constitutive relations, excitation forces or influences of physical fields $[6,7]$. A special class of beam structures is so-called nonlocal beams, where the 
nonlocal elasticity constitutive equation is employed to consider the small-scale effects [8]. Such nonlocal beams are usually referred to in the literature as nanobeams due to the nano-scale dimensions of structures.

Nonlocal theory was applied for the static [9], dynamic [10] and instability analysis [11] of nanobeams with or without considered surface effects. In addition, static, buckling and dynamic behaviour of nonlocal and higher order gradient beams was investigated and discussed by Eltaher et al. [12]. In more recent studies, previous theoretical observations regarding the size dependent theories for nanoberams were applied in the analysis of more complex models of nanosensors [13], perforated beams [14], systems with piezoelectric [15] and magneto-thermal [16, 17] effects.

It is well-known that effects of external medium surrounding beam structures can be represented by different types of elastic foundation or coupling layer models [18]. Foundation models can be extended to account nonlinear effects using energy equivalent model and shearing layer properties $[19,20]$. Such model was used by Eltaher et al. [21] in buckling, postbuckling and dynamic analysis of a beam resting on nonlinear elastic foundation. Emam et al. investigated postbuckling of multilayer imperfect nanobeam under a prestress load [22]. Moreover, a novel numerical procedure was suggested by Mohamed et al. [23] to predict nonlinear free and steady state forced vibrations of curved beams surrounded by nonlinear elastic foundation with cubic nonlinearity and shearing layer. However, in most application examples linear elastic/viscoelastic foundation models can satisfy requirements for describing the effects of surrounding medium on observed structural element, which is the case observed in this study.

Vibration damping can be observed in nanobeam models to account for the internal or external sources of dissipation [24]. Recently, nonlocal elastic and fractional viscoelastic models of nanobeams have been introduced [25, 26]. Later, Ansari et al. [27] utilized such a model to study the geometrically nonlinear vibration of fractional viscoelastic nanobeams by using numerical methods. In [28], this methodology was extended to study linear and nonlinear vibrations of fractional viscoelastic Timoshenko nanobeams considering surface energy effect. However, multiple scales (MS) perturbation method was employed by Eyebe et al. [29] to study the steady-state frequency response of a nonlinear nanobeam system resting on the fractional-order viscoelastic Winkler-Pasternak foundation. Farhatnia et al. [30] studied buckling of FG plate resting on Pasternak elastic layer using differential transform method. Jha and Dasgupta [31] used the approximate averaging method to found the analytical solution of the Duffing-type differential equation obtained for the nonlinear fractionally damped nanobeam structure. The authors adopted the Galerkin method to discretize the governing equation of the system. The overview of the papers using multiple time scales technique for finding the solution of nonlinear fractional-order differential equations can be found in [32].

The main advantage of harmonic balance techniques is that they can be employed to find the periodic solutions of strongly nonlinear systems without introducing a small parameter like in perturbation techniques [33]. Shen et al. [34] investigated the Mathieu-Duffing oscillator by the incremental harmonic balance (IHB) method and determined the stability of the periodic solution using the Floquet theory. Later, this method was extended to study the fractional-order nonlinear Duffing [35] and forced Mathieu-Duffing type [36] of equations, where the incremental harmonic balance method and Galerkin procedure is used to transform the fractional nonlinear differential equation into an algebraic nonlinear system of equations. The results are verified with the averaging method. Sourani et al. [37] studied the dynamic stability of the 
Euler-Bernoulli nanobeam under the time-dependent axial loading using the nonlocal strain gradient theory and taking into account surface stress effects and thermal gradient. The geometric nonlinearity is considered through the Von Kármán strain-displacement relation. The authors applied Bolotin and Incremental Harmonic Balance (IHB) methods to study the impact of different parameters on the dynamic stability. Moreover, in [38] the authors studied a nonlinear model of a nanobeam resting on the viscoelastic foundation and under the influence of axial dynamic load. In this paper, a multiple scales method was applied to obtain the nonlinear frequency equation and linear instability regions. Besides, the incremental harmonic balance method was employed to investigate nonlinear instability regions.

Some authors studied the more complex nonlinear structural vibration problems with fractional-order rheological models. Lewandowski and Wielentejczyk $[39,40]$ studied the problem of nonlinear vibration of harmonically excited beams by using the fractional-order Zener type rheological models to describe the viscoelastic material behaviour of the beams. In these papers, the solution of the system of nonlinear differential equations is sought by the harmonic balance and continuation method. The stability of steadystate solutions is verified by using the averaging method and Floquet theory as well.

This study aims to employ the multiple time scales perturbation and incremental harmonic balance techniques to study the frequency response of a nanobeam system resting on the fractional visco-Pasternak type foundation. The single-mode Galerkin method is used to discretize the governing equation and obtain the nonlinear response for the fractional-order forced Mathieu-Duffing equation. The results are verified by the comparison of amplitude-frequency curves from the multiple scales and incremental harmonic balance methods obtained for the superharmonic resonance conditions of order two and three. The parametric study is performed for both, weak nonlinear forced oscillations using the perturbation method and strong nonlinear case by the IHB method.

\section{Preliminaries}

Constitutive equations of the nonlocal elasticity theory are based on the assumption that the stress at some point is a function of strains at all other points of the elastic body. This theory was initially introduced by Eringen [41], which in the last two decades shown to be very useful in describing the small-scale effects in nanostructures. Initially, Eringen [41] proposed the integral form of the constitutive equation to include the nonlocal effects into the model. However, this problem was simplified by deriving the differential form of the nonlocal constitutive equation, as given in $[8,42]$. In this paper, we adopt the following form of nonlocal constitutive relation

$$
\begin{gathered}
\sigma_{x x}-\mu \frac{\partial^{2} \sigma_{x x}}{\partial x^{2}}=E \varepsilon_{x x}, \\
\tau_{x z}-\mu \frac{\partial^{2} \tau_{x z}}{\partial x^{2}}=2 G \varepsilon_{x z},
\end{gathered}
$$

with $E$ and $G$ denoting the elastic and shear modulus, respectively, $\mu=\left(e_{0} a\right)^{2}$ is the nonlocal parameter with $a$ denoting the internal characteristic length (lattice parameter, granular, etc.), $e_{0}$ is the constant 
specific to each material and $\sigma_{x x}$ and $\tau_{x z}$ are the nonlocal normal and shear stresses, respectively. In this study, we adopted the Euler-Bernoulli beam theory and therefore Eq. (2) is omitted in further analysis.

To represent the influence of the fractional visco-Pasternak foundation on the nanobeam, we will employ the phenomenological model of this foundation through the fractional-order relationship between restoring force and displacement of foundation acting on the nanobeam as one-dimensional structure. This relation is adopted from [44] and it takes the form

$$
\vartheta(x)=\bar{\delta} D_{t}^{\alpha}(w)+\bar{G}_{p} D_{t}^{\alpha}\left(\frac{\partial^{2} w}{\partial x^{2}}\right),
$$

where $\bar{\delta}$ and $\bar{G}_{p}$ are the coefficients of the fractional visco-Pasternak foundation and $D_{t}^{\alpha}=d^{\alpha} / d t^{\alpha}$ is the operator of the fractional-order derivative [43]. Here, two well-known definitions of the fractional derivative will be employed [47]. The Riemann-Liouville definition of fractional-order derivative is used in Eq. (2) for the case when the multiple time scales method is applied. Next, instead of the previous one, the Caputo definition of fractional-order derivative is used to derive the relations for the incremental harmonic balance method. It should be noted that the model adopted here is similar to those presented in [29] but yet with the slightly different equation for the fractional visco-Pasternak foundation and numerical analysis for the strongly nonlinear case.

\section{Problem definition}

\subsection{Derivation of the governing equation}

The governing equation for the forced vibration of a nanobeam resting on the fractional visco-Pasternak foundation can be derived based on the model presented in Fig. 1. It should be noted that a nonlocal beam model could represent nanostructures such as carbon nanotube. Bearing in mind the perspective application of carbon nanotubes as actuators [52, 53], nonlinear analysis of such structures based on reliable models accounting size effects is important research subject. Besides, it is important to prescribe proper boundary conditions to analyse the free or forced vibration of a nanobeam structure based on the end conditions of a carbon nanotube depending on the number of fixed layers of atoms in the lattice (e.g. see [48]). If only one layer of atoms is fixed at both ends of carbon nanotube, we can use simply supported (S-S) boundary conditions in the mechanical model, and if several layers of atoms are fixed, we can use boundary conditions of clamped-clamped (C-C) nanobeam. Let us define the parameters of the presented model where $L$ is the length of the nanobeam, $\rho$ is the density, $A$ is the cross-sectional area of homogenous nanobeam. Parameters of the fractional visco-Pasternak foundation are given in Eq. (3).

Based on the Euler-Bernoulli beam theory and von Kármán nonlinear deformation, we can write the strain displacement relation in the form

$$
\varepsilon_{x x}=\frac{\partial \bar{u}_{x}}{\partial x}+\frac{1}{2}\left(\frac{\partial w}{\partial x}\right)^{2}=\frac{\partial u}{\partial x}-z \frac{\partial^{2} w}{\partial x^{2}}+\frac{1}{2}\left(\frac{\partial w}{\partial x}\right)^{2},
$$

where $u$ and $w$ are displacements in the axial and transverse directions of the nanobeam and $\varepsilon_{x x}$ is the deformation in the $x$ direction. 
a) S-s

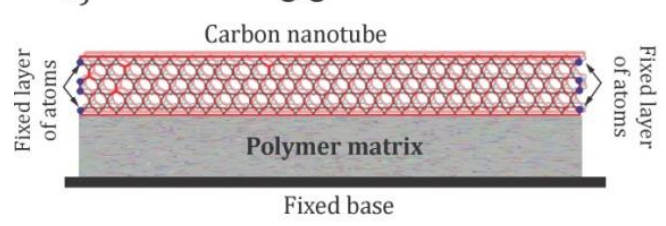

b)

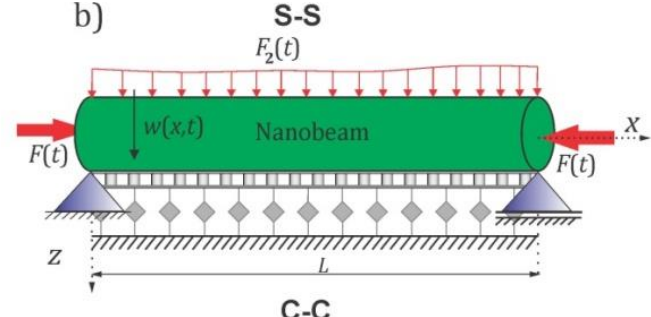

C-C

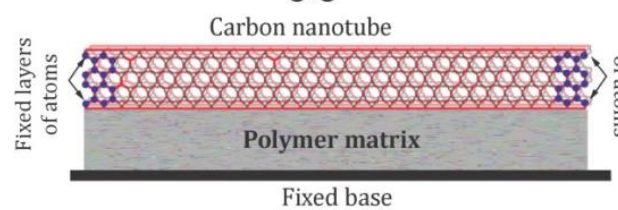

Fixed base

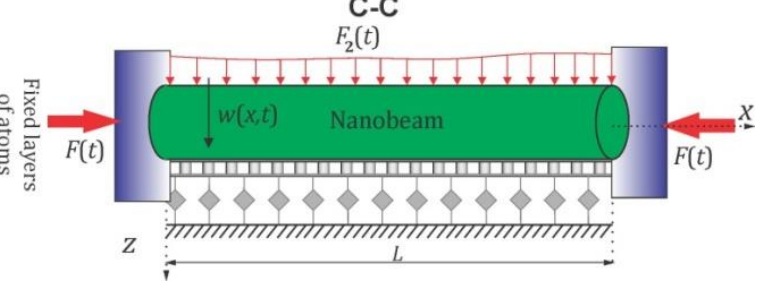

Fig. 1 Illustration of a nanobeam on the fractional visco-Pasternak foundation

Following the methodology from [46] and [38] we can write dynamic equilibrium equations of the element of the vibrating nanobeam as

$$
\begin{aligned}
\frac{\partial V}{\partial x}+f(x, t)-\vartheta(x)+\frac{\partial}{\partial x}\left(S \frac{\partial w}{\partial x}\right)+\frac{1}{2}\left(\frac{\partial w}{\partial x}\right)^{2}=\rho A \frac{\partial^{2} w}{\partial t^{2}}, \\
\frac{\partial S}{\partial x}=\rho A \frac{\partial^{2} u}{\partial t^{2}} \\
\frac{\partial M}{\partial x}=V,
\end{aligned}
$$

where $V=\int_{A} \tau_{x z} d A$ is the transverse force, $S=\int_{A} \sigma_{x x} d A$ is the axial force, $M=\int_{A} z \sigma_{x x} d A$ are the bending moment and transverse excitation force given as $f(x, t)=\bar{F}_{2} \cos \Omega_{2} t$.

To derive the governing equation we need first to determine the axial force $S$. By replacing Eq. (4) into the nonlocal constitutive equation (1) and taking into account equations for the axial and transverse forces we obtain the following equation for the nonlocal axial force

$$
S-\mu \frac{\partial^{2} S}{\partial x^{2}}=E A\left(\frac{\partial u}{\partial x}+\frac{1}{2}\left(\frac{\partial w}{\partial x}\right)^{2}\right)
$$

and the nonlocal bending moment

$$
M-\mu \frac{\partial^{2} M}{\partial x^{2}}=-E I \frac{\partial^{2} w}{\partial x^{2}} .
$$

We further neglect the inertia term $\rho A \frac{\partial^{2} u}{\partial t^{2}}$ in (6) and the axial force becomes equal to the sum of forces acting in the axial direction and forces from the geometric nonlinearity, which yields

$$
\frac{\partial u}{\partial x}=\frac{S}{E A}-\frac{1}{2}\left(\frac{\partial w}{\partial x}\right)^{2} \text {. }
$$

Integrating Eq. (10) along the length of the nanobeam and taking into account boundary conditions $u(0, t)=$ 0 and $u(L, t)=\Delta L$, where $\Delta L=F L / E A$ (e.g. see [46]), we obtain the equation for the axial force as

$$
S=F+\frac{E A}{2 L} \int_{0}^{L} \frac{1}{2}\left(\frac{\partial w}{\partial x}\right)^{2} d x .
$$

Let us assume the axial dynamic force as $F=-\left(\bar{F}_{0}+\bar{F}_{1} \cos \Omega_{1} t\right)$, where $\bar{F}_{0}$ is the amplitude of static load while $\bar{F}_{1}$ is the amplitude of the dynamic force of the frequency $\Omega_{1}$. Finally, based on previous equations one 
can derive the nonlinear fractional-order partial differential equation of motion of the nanobeam resting on the fractional visco-Pasternak foundation as

$$
\begin{gathered}
\rho A \frac{\partial^{2} w}{\partial t^{2}}+D_{t}^{\alpha} w \bar{\delta}+D_{t}^{\alpha}\left(\frac{\partial^{2} w}{\partial x^{2}}\right) \bar{G}_{p}+\left(\bar{F}_{0}+\bar{F}_{1} \cos \Omega_{1} t\right) \frac{\partial^{2} w}{\partial x^{2}} \\
-\frac{E A}{2 L} \frac{\partial^{2} w}{\partial x^{2}} \int_{0}^{L} \frac{1}{2}\left(\frac{\partial w}{\partial x}\right)^{2} d x+E I \frac{\partial^{4} w}{\partial x^{4}}-\mu\left[\rho A \frac{\partial^{4} w}{\partial t^{2} \partial x^{2}}+D_{t}^{\alpha} \frac{\partial^{2} w}{\partial x^{2}} \bar{\delta}+D_{t}^{\alpha}\left(\frac{\partial^{4} w}{\partial x^{4}}\right) \bar{G}_{p}\right. \\
\left.+\left(\bar{F}_{0}+\bar{F}_{1} \cos \Omega_{1} t\right) \frac{\partial^{4} w}{\partial x^{4}}-\frac{E A}{2 L} \frac{\partial^{4} w}{\partial x^{4}} \int_{0}^{L} \frac{1}{2}\left(\frac{\partial w}{\partial x}\right)^{2} d x\right]=\bar{F}_{2} \cos \Omega_{2} t
\end{gathered}
$$

In follow, we will observe only the dimensionless form of this equation given as

$$
\begin{gathered}
\frac{\partial^{2} \bar{w}}{\partial \tau^{2}}+D_{\tau}^{\alpha} \bar{w} \delta+D_{\tau}^{\alpha}\left(\frac{\partial^{2} \bar{w}}{\partial \bar{x}^{2}}\right) G_{p}+\left(F_{0}+F_{1} \cos \bar{\Omega}_{1} \tau\right) \frac{\partial^{2} \bar{w}}{\partial \bar{x}^{2}} \\
-R \frac{\partial^{2} \bar{w}}{\partial \bar{x}^{2}} \int_{0}^{1} \frac{1}{2}\left(\frac{\partial \bar{w}}{\partial \bar{x}}\right)^{2} d \bar{x}+\frac{\partial^{4} \bar{w}}{\partial \bar{x}^{4}}-\eta^{2}\left[\frac{\partial^{4} \bar{w}}{\partial \tau^{2} \partial \bar{x}^{2}}+D_{\tau}^{\alpha} \frac{\partial^{2} \bar{w}}{\partial \bar{x}^{2}} \delta+D_{\tau}^{\alpha}\left(\frac{\partial^{4} \bar{w}}{\partial \bar{x}^{4}}\right) G_{p}\right. \\
\left.+\left(F_{0}+F_{1} \cos \bar{\Omega}_{1} \tau\right) \frac{\partial^{4} \bar{w}}{\partial \bar{x}^{4}}-R \frac{\partial^{4} \bar{w}}{\partial \bar{x}^{4}} \int_{0}^{1} \frac{1}{2}\left(\frac{\partial \bar{w}}{\partial \bar{x}}\right)^{2} d \bar{x}\right]=F_{2} \cos \bar{\Omega}_{2} \tau
\end{gathered}
$$

with dimensionless parameters

$$
\begin{gathered}
\bar{w}=\frac{w}{L}, \bar{x}=\frac{x}{L}, \eta^{2}=\frac{\mu}{L}, c=\left(\frac{E I}{L^{4} \rho A}\right)^{1 / 2}, \tau=t c, \tau^{\alpha}=t^{\alpha} c^{\alpha}, \quad \delta=\bar{\delta} \frac{L^{4}}{E I} c^{\alpha}, \\
G_{p}=\bar{G}_{p} \frac{L^{2}}{E I} c^{\alpha}, R=\frac{L A}{2 I}, \bar{F}_{0}=\frac{F_{0} L^{2}}{E I}, \quad F_{1}=\frac{\bar{F}_{1} L^{2}}{E I}, \quad F_{2}=\frac{\bar{F}_{2} L^{3}}{E I} .
\end{gathered}
$$

\section{2 Problem solution}

In the general case, closed-form analytical solutions of nonlinear differential equations are difficult to find. Therefore, various numerical and approximate methods are used in the literature to find the solution to this problem. For the discretization of nonlinear partial differential equations, one may use whether Ritz [51] or the Galerkin method with linear mode shape functions as trial functions. Nayfeh et al. [45] have demonstrated that the single-mode Galerkin method yields satisfying results when the third-order nonlinear equation is considered but not for the equations with the second-order nonlinearity. Here, we use the single-mode Galerkin discretization since only the third-order nonlinear term is present in the nonlinear fractional-order differential equation. Accordingly, we assume the solution of Eq. (13) in the following form

$$
\bar{w}(\bar{x}, \tau)=\phi_{n}(\bar{x}) q_{n}(\tau)
$$

where $\phi_{n}(\bar{x})$ is the amplitude function, $q_{n}(\tau)$ is the time function and $n=1,2, \ldots$ is the mode number. By replacing Eq. (15) into Eq. (13) we obtain the following nonlinear fractional-order differential equation

$$
\ddot{q}+\bar{\gamma}_{12} D_{\tau}^{\alpha} q+\left(\omega_{n}^{2}-\bar{\gamma}_{3} F_{1} \cos \bar{\Omega}_{1} \tau\right) q+\bar{\gamma}_{4} q^{3}=\tilde{f} \cos \bar{\Omega}_{2} \tau,
$$

where parameters are given as

$$
\begin{gathered}
\omega_{n}=\left[\frac{b_{3}-\left(b_{2}-\eta^{2} b_{3}\right) F_{0}}{b_{1}-\eta^{2} b_{2}}\right]^{1 / 2}, \quad \bar{\gamma}_{12}=\delta-G_{p} \frac{b_{2}-\eta^{2} b_{3}}{b_{1}-\eta^{2} b_{2}}, \quad \bar{\gamma}_{3}=\frac{-b_{2}+\eta^{2} b_{3}}{b_{1}-\eta^{2} b_{2}}, \\
\bar{\gamma}_{4}=R b_{4} \bar{\gamma}_{3}, \quad \tilde{f}=\frac{F_{2} b_{0}}{b_{1}-\eta^{2} b_{2}},
\end{gathered}
$$

Coefficients $b_{0}-b_{4}$ are calculated as 


$$
\left\{b_{0}, b_{1}, b_{2}, b_{3}, b_{4}\right\}=\int_{0}^{1}\left\{\phi, \phi^{2}, \phi^{\prime \prime} \phi, \phi^{I V} \phi,\left(\phi^{\prime}\right)^{2}\right\} d \bar{x} .
$$

For $\phi_{n}(\bar{x})$ we choose the linear mode shape functions corresponding to simply supported and clampedclamped boundary conditions. The linear mode shape functions are normalized to satisfy the following condition

$$
\int_{0}^{1} \phi_{n}(\bar{x}) \phi_{j}(\bar{x}) d \bar{x}=\delta_{n j}
$$

where $\delta_{n j}$ is the Kronecker delta for $n, j=1,2, \ldots, \infty$. Therefore, for the simply supported nanobeam, we use

$$
\phi_{n}(\bar{x})=C_{n} \sin k_{n} \bar{x},
$$

while for the clamped-clamped nanobeam, we have

$$
\phi_{n}(\bar{x})=C_{n}\left\{\cosh k_{n} \bar{x}-\cos k_{n} \bar{x}-\frac{\cosh k_{n} \bar{x}-\cos k_{n} \bar{x}}{\sinh k_{n} \bar{x}-\sin k_{n} \bar{x}}\left[\sinh k_{n} \bar{x}-\sin k_{n} \bar{x}\right]\right\},
$$

where $C_{n}$ are constants to be determined from the boundary conditions and $k_{n}$ are eigenvalues that are well known from the linear vibration analysis.

\section{3 Multiple scales method}

Above Eq. (16) is known as the forced Mathieu-Duffing fractional-order differential equation, which we can express in terms of small parameter $\varepsilon$ as

$$
\ddot{q}+\varepsilon \gamma_{12} D_{\tau}^{\alpha} q+\left(\omega_{n}^{2}-\varepsilon \gamma_{3} F_{1} \cos \bar{\Omega}_{1} \tau\right) q+\varepsilon \gamma_{4} q^{3}=\tilde{f} \cos \bar{\Omega}_{2} \tau,
$$

where we introduce new parameters as $\bar{\gamma}_{12}=\varepsilon \gamma_{12}, \bar{\gamma}_{3}=\varepsilon \gamma_{3}$ and $\bar{\gamma}_{4}=\varepsilon \gamma_{4}$ with $\varepsilon$ denoting the small time scale parameter and $D^{\alpha}$ denoting, in this case, the operator of Riemann-Liouville fractional derivative (e.g. see [47]). The small parameter $\varepsilon$ is introduced in front of the fractional and nonlinear terms to have weak damping and weak nonlinearity. It should be noted that forcing term in Eq. (22) is of order one (also known as hard forcing) which will help us to study secondary resonances in the system using the perturbation analysis of the first order. According to [49], forcing of order $\varepsilon$ would indicate a primary resonance that is the same as in the Duffing equation.

For the solution of Eq. (22) we will use the multiple scales method by assuming the solution in the following form

$$
q\left(\mathrm{~T}_{0}, \mathrm{~T}_{1}, \varepsilon\right)=q_{0}\left(\mathrm{~T}_{0}, \mathrm{~T}_{1}\right)+\varepsilon q_{1}\left(\mathrm{~T}_{0}, \mathrm{~T}_{1}\right)+\cdots,
$$

where $\mathrm{T}_{0}=\tau$ is fast and $\mathrm{T}_{1}=\varepsilon \tau$ is a slow time scale. Here we will analyse the case of combined parametric and external resonance condition, where we adopt $\bar{\Omega}_{1}=\bar{\Omega}_{2}=\bar{\Omega}$. This will be later used to analyze the system for superharmonic resonance conditions.

First, we will define the time derivatives as

$$
\begin{gathered}
\frac{d}{d \tau}=D_{0}+\varepsilon D_{1}+O\left(\varepsilon^{2}\right), \\
\frac{d^{2}}{d \tau^{2}}=D_{0}^{2}+2 \varepsilon D_{0} D_{1}+O\left(\varepsilon^{2}\right),
\end{gathered}
$$




$$
D_{\tau}^{\alpha}=D_{0+}^{\alpha}-\varepsilon \alpha D_{0+}^{\alpha-1} D_{1}+\cdots,
$$

where $D_{n}=\frac{\partial}{\partial \mathrm{T}_{n}},(n=0,1,2, \ldots)$ and $D_{n+}^{\alpha-n}=\frac{\partial^{\alpha-n}}{\partial \mathrm{T}_{n+}^{\alpha-n}},(n=0,1,2, \ldots)$ are classical and Riemann-Liouville's fractional derivative for new time scales, e.g. see [32].

For the fractional derivative of the exponential function [32], we will adopt the following relationship that is restricted to the first and second-order approximations as

$$
D_{0+}^{\alpha} e^{i \omega \tau}=(i \omega)^{\alpha} e^{i \omega \tau}
$$

where $i$ is the imaginary unit. Replacing Eqs. (23)-(26) into Eq. (22) and then simplifying and extracting coefficients of $\varepsilon^{0}$ and $\varepsilon^{1}$ we get the following equations

$$
\begin{gathered}
\varepsilon^{0}: D_{0}^{2} q_{0}+\omega_{n}^{2} q_{0}=\tilde{f} \cos \bar{\Omega} \tau, \\
\varepsilon^{1}: D_{0}^{2} q_{1}+\omega_{n}^{2} q_{1}=-2 D_{0} D_{1} q_{0}-\gamma_{12} D_{0+}^{\alpha} q_{0}-\gamma_{4} q_{0}^{3}+\frac{1}{2} \gamma_{3} \bar{F}_{1}\left(e^{i \bar{\Omega} \mathrm{T}_{0}}+e^{-i \bar{\Omega} \mathrm{T}_{0}}\right) q_{0} .
\end{gathered}
$$

The solution of Eq. (28) is sought in the form

$$
q_{0}=\mathrm{A}\left(\mathrm{T}_{1}\right) e^{i \omega_{n} \mathrm{~T}_{0}}+\Lambda e^{i \bar{\Omega} \mathrm{T}_{0}}+\text { c.c., }
$$

where $\mathrm{A}$ is a complex function in terms of slow time scale, c.c. are complex conjugate terms and $\Lambda$ is defined as

$$
\Lambda=\frac{\tilde{f}}{2\left(\omega_{n}^{2}-\bar{\Omega}^{2}\right)}
$$

\subsubsection{Superharmonic resonance $2 \bar{\Omega} \approx \omega_{0 n}$}

Let us now consider that $2 \bar{\Omega}=\omega_{n}+\varepsilon \sigma$, where $\sigma$ is the detuning parameter. After replacing the solution for $q_{0}$ Eq. (30) into Eq. (29) and equating the secular terms $e^{i \omega_{n} \mathrm{~T}_{0}}$ with zero, we obtain the solvability conditions for the superharmonic resonance as

$$
-2 i \omega_{n} A^{\prime}-\gamma_{12} A\left(i \omega_{n}\right)^{\alpha}-6 \gamma_{4} A \Lambda^{2}-3 \gamma_{4} A^{2} \bar{A}+\frac{1}{2} \gamma_{3} \Lambda F_{1} e^{i \sigma \mathrm{T}_{1}}=0,
$$

where $A^{\prime}=D_{1} A$. If we take that $\mathrm{A}=1 / 2 a e^{i \varphi}$, where $a$ and $\varphi$ are the amplitude and phase of the nonlinear system, respectively and replace it in Eq. (32), separation of the real and the imaginary parts leads to

$$
\begin{gathered}
\omega_{n} \varphi^{\prime}-3 \gamma_{4} \Lambda^{2} a-\frac{3}{8} \gamma_{4} a^{3}-\frac{1}{2} \gamma_{12} a \omega_{n}^{\alpha} \cos \frac{\alpha \pi}{2}+\frac{1}{4} \gamma_{3} \Lambda F_{1} \cos \theta=0, \\
-\omega_{n} a^{\prime}-\frac{1}{2} \gamma_{12} a \omega_{n}^{\alpha} \sin \frac{\alpha \pi}{2}+\frac{1}{2} \gamma_{3} \Lambda F_{1} \sin \theta=0,
\end{gathered}
$$

with $\theta=\sigma \mathrm{T}_{1}-\varphi$ denoting the new phase angle. A relationship between the response amplitude and the detuning parameter can be obtained by using the following steady-state conditions $a^{\prime}=0, \theta^{\prime}=0$, wherefrom Eq. (33) and (34) we have

$$
\begin{gathered}
\frac{1}{2} \gamma_{3} \frac{\Lambda F_{1}}{a \omega_{n}} \cos \theta=-\sigma+3 \gamma_{4} \frac{\Lambda^{2}}{\omega_{n}}+\frac{3}{8} \frac{\gamma_{4}}{\omega_{n}} a^{2}+\gamma_{12} \omega_{n}^{\alpha-1} \cos \frac{\alpha \pi}{2}, \\
\frac{1}{2} \gamma_{3} \frac{\Lambda F_{1}}{a \omega_{n}} \sin \theta=\frac{1}{2} \gamma_{12} \omega_{n}^{\alpha-1} \sin \frac{\alpha \pi}{2} .
\end{gathered}
$$

After some algebra over Eqs. (35) and (36) one obtains the polynomial equation as

$$
\sigma^{2}-2 K \sigma+M=0,
$$

where $K$ and $M$ are given as 


$$
\begin{gathered}
K=3 \gamma_{4} \frac{\Lambda^{2}}{\omega_{n}}+\frac{3}{8} \frac{\gamma_{4}}{\omega_{n}} a^{2}+\frac{1}{2} \gamma_{12} \omega_{n}^{\alpha-1} \cos \frac{\alpha \pi}{2}, \\
M=\left[3 \gamma_{4} \frac{\Lambda^{2}}{\omega_{n}}+\frac{3}{8} \frac{\gamma_{4}}{\omega_{n}} a^{2}+\frac{1}{2} \gamma_{12} \omega_{n}^{\alpha-1} \cos \frac{\alpha \pi}{2}\right]^{2}+\left[\frac{1}{2} \gamma_{12} \omega_{n}^{\alpha-1} \sin \frac{\alpha \pi}{2}\right]^{2}-\left[\frac{1}{2} \gamma_{3} \frac{\Lambda F_{1}}{a \omega_{n}}\right]^{2},
\end{gathered}
$$

wherefrom we can finally obtain the relationship for the amplitude-frequency curves as

$$
\sigma_{1 / 2}=K \pm \sqrt{K^{2}-M}
$$

One can notice that all the parameters contribute to the appearance of superharmonic resonance of order $1 / 2$ i.e. we have interaction of the nonlinear term, fractional-order term, parametric and external excitation terms.

\subsubsection{Superharmonic resonance $3 \bar{\Omega} \approx \omega_{n}$}

Let us now consider the case when $3 \bar{\Omega}=\omega_{n}+\varepsilon \sigma$, where $\sigma$ is again the detuning parameter. After replacing the solution for $q_{0}$ Eq. (30) into Eq. (29) and equating the secular terms $e^{i \omega_{n} \mathrm{~T}_{0}}$ with zero, we obtain the solvability conditions as

$$
-2 i \omega_{n} \mathrm{~A}^{\prime}-\gamma_{12} A\left(i \omega_{n}\right)^{\alpha}-6 \gamma_{4} A \Lambda^{2}-3 \gamma_{4} A^{2} \bar{A}-\gamma_{4} \Lambda^{3} e^{i \sigma \mathrm{T}_{1}}=0,
$$

Replacing again $\mathrm{A}=1 / 2 a e^{i \varphi}$ into Eq. (42) and separating real and imaginary parts yields

$$
\begin{gathered}
\omega_{n} a \varphi^{\prime}-\frac{1}{2} \gamma_{12} a \omega_{n}^{\alpha} \cos \frac{\alpha \pi}{2}-\frac{3}{8} \gamma_{4} a^{3}-3 \gamma_{4} \Lambda^{2} a-\gamma_{4} \Lambda^{3} \cos \theta=0, \\
-\omega_{n} a^{\prime}-\frac{1}{2} \gamma_{12} a \omega_{n}^{\alpha} \sin \frac{\alpha \pi}{2}-\gamma_{4} \Lambda^{3} \sin \theta=0,
\end{gathered}
$$

with $\theta=\sigma \mathrm{T}_{1}-\varphi$ denoting the new phase angle. Using the same steady-state condition as in the previous case we obtain

$$
\begin{gathered}
\frac{\gamma_{4} \Lambda^{3}}{\omega_{n} a} \cos \theta=\sigma-\frac{1}{2} \gamma_{12} \omega_{0 n}^{\alpha-1} \cos \frac{\alpha \pi}{2}-\frac{3}{8} \frac{\gamma_{4}}{\omega_{n}} a^{2}-3 \frac{\gamma_{4} \Lambda^{2}}{\omega_{n}}, \\
\frac{\gamma_{4} \Lambda^{3}}{\omega_{n} a} \sin \theta=-\gamma_{12} \omega_{n}^{\alpha-1} \sin \frac{\alpha \pi}{2} .
\end{gathered}
$$

After some algebra over Eqs. (44) and (45) one obtains the polynomial equation for the present superharmonic resonance case as

$$
\sigma^{2}-2 K_{1} \sigma+M_{1}=0
$$

where $K_{1}$ and $M_{1}$ are given as

$$
\begin{gathered}
K_{1}=3 \gamma_{4} \frac{\Lambda^{2}}{\omega_{n}}+\frac{3}{8} \frac{\gamma_{4}}{\omega_{n}} a^{2}+\frac{1}{2} \gamma_{12} \omega_{n}^{\alpha-1} \cos \frac{\alpha \pi}{2}, \\
M_{1}=\left[3 \gamma_{4} \frac{\Lambda^{2}}{\omega_{n}}+\frac{3}{8} \frac{\gamma_{4}}{\omega_{n}} a^{2}+\frac{1}{2} \gamma_{12} \omega_{n}^{\alpha-1} \cos \frac{\alpha \pi}{2}\right]^{2}+\left[\frac{1}{2} \gamma_{12} \omega_{n}^{\alpha-1} \sin \frac{\alpha \pi}{2}\right]^{2}-\left[\frac{\gamma_{4} \Lambda^{3}}{a \omega_{n}}\right]^{2},
\end{gathered}
$$

wherefrom we can finally obtain the relationship for the amplitude-frequency curves as

$$
\sigma_{1 / 2}=K_{1} \pm \sqrt{K_{1}^{2}-M_{1}}
$$

In this case, the parametric excitation term does not contribute to superharmonic resonance of order $1 / 3$ for the first-order perturbation analysis while we have interaction of the nonlinear and external forcing terms. In [49], the authors revealed the existence of superharmonic resonance in the higher-order 
perturbation analysis, even in the absence of nonlinearity. It is also concluded that the equivalent parametrically and the directly excited linear system can also exhibit superharmonic resonances. However, multiple scales analysis requires the introduction of the small parameter and therefore analysis is limited to weakly nonlinear and weakly damping cases. This problem can be overcome by the incremental harmonic balance method analysis that can be used easily to study periodic responses of strongly nonlinear systems without introducing the small parameter.

\section{4 Incremental harmonic balance method}

Here, we suggest another semi-analytical approach based on the incremental harmonic balance method for finding the steady-state response of the system. First, one should introduce a new time scale $\bar{\tau}=\bar{\Omega} \tau$ into Eq. (22) to obtain the system of nonlinear ordinary differential equations in the following form

$$
\bar{\Omega}^{2} q^{\prime \prime}+\varepsilon \gamma_{12} \bar{\Omega}^{\alpha} D_{\bar{\tau}}^{\alpha} q+\left(\omega_{n}^{2}-\varepsilon \gamma_{3} F_{1} \cos \bar{\tau}\right) q+\varepsilon \gamma_{4} q^{3}=\tilde{f} \cos \bar{\tau},
$$

Now, we assume that $D^{\alpha}$ represents the operator of the Caputo fractional derivative (e.g. see [47]). Let us also assume that the neighboring state of the periodic solution can be expressed by adding the corresponding increments. For some initial guess $q_{0}$ and $\bar{\Omega}_{0}$ of the steady-state modal amplitude, a neighbouring state of motion can be expressed in the following form

$$
q=q_{0}+\Delta q, \quad \bar{\Omega}=\bar{\Omega}_{0}+\Delta \bar{\Omega} .
$$

Substituting Eq. (51) into Eq. (50) and neglecting higher-order terms we obtain a linearized incremental relation given as

$$
\begin{gathered}
\bar{\Omega}_{0}^{2} \Delta q^{\prime \prime}+\varepsilon \gamma_{12} \bar{\Omega}_{0}^{\alpha} D_{\bar{\tau}}^{\alpha} \Delta q+\left(\omega_{n}^{2}-\varepsilon \gamma_{3} F_{1} \cos \bar{\tau}\right) \Delta q+3 \varepsilon \gamma_{4} q_{0}^{2} \Delta \\
=R-\left\{2 \bar{\Omega}_{0} q_{0}^{\prime \prime}+\varepsilon \gamma_{12} \alpha \bar{\Omega}_{0}^{\alpha-1} D_{\bar{\tau}}^{\alpha} q_{0}\right\} \Delta \bar{\Omega},
\end{gathered}
$$

where $R$ is the residual term given as

$$
R=\bar{\Omega}_{0}^{2} q_{0}^{\prime \prime}-\varepsilon \gamma_{12} \bar{\Omega}_{0}^{\alpha} D_{\bar{\tau}}^{\alpha} q_{0}-\left(\omega_{n}^{2}-\varepsilon \gamma_{3} F_{1} \cos \bar{\tau}\right) q_{0}-\varepsilon \gamma_{4} q_{0}^{3}+\tilde{f} \cos \bar{\tau},
$$

which is equal to zero $R=0$ if $q_{0}$ is the exact solution. Next, to obtain the periodic solutions of the forced Mathieu-Duffing fractional-order differential equation, $q_{0}, \Delta q, \bar{\Omega}_{0}$ and $\Delta \bar{\Omega}$ are expanded as a finite Fourier series of $N$ terms as

$$
\begin{array}{r}
q_{0}=a_{0}+\sum_{n=1}^{N}\left[a_{n} \cos (n \bar{\tau})+b_{n} \sin (n \bar{\tau})\right]=\boldsymbol{C} \cdot \boldsymbol{A}_{\mathbf{0}}, \\
\Delta q=\Delta a_{0}+\sum_{n=1}^{N}\left[\Delta a_{n} \cos (n \bar{\tau})+\Delta b_{n} \sin (n \bar{\tau})\right]=\boldsymbol{C} \cdot \Delta \boldsymbol{A},
\end{array}
$$

where

$$
\begin{gathered}
\boldsymbol{C}=\left[\begin{array}{cccccccccc}
\cos \bar{\tau} \cos 2 \bar{\tau} & \cos 3 \bar{\tau} & \ldots & \cos N_{h} \bar{\tau} & \sin \bar{\tau} \sin 2 \bar{\tau} & \sin \bar{\tau} & \ldots & \sin N_{h} \bar{\tau}
\end{array}\right], \\
\boldsymbol{A}_{\mathbf{0}}=\left[\begin{array}{lllllllllll}
a_{0} & a_{1} & a_{2} & \ldots & a_{N} & b_{1} & b_{2} & b_{3} & \ldots & b_{N}
\end{array}\right]^{T}, \\
\Delta \boldsymbol{A}=\left[\begin{array}{llllllllll}
\Delta a_{0} & \Delta a_{1} & \Delta a_{2} & \ldots & \Delta a_{N} & \Delta b_{1} & \Delta b_{2} & \Delta b_{3} & \ldots & \Delta b_{N}
\end{array}\right]^{T} .
\end{gathered}
$$

After considering Eqs. (54) - (58) into the Eq. (52), we apply the Galerkin procedure as presented in [35]. Considering the fact, that fractional-order derivative is an aperiodic function, in the integration procedure we select the time terminal $T=\infty$ and average the integration results for the fractional derivative. Based on this procedure, for the periodic function, we select the time terminal as $T=2 \pi$ to obtain the following system of equations 


$$
\begin{gathered}
\frac{1}{2 \pi} \int_{0}^{2 \pi}(\delta \Delta q)\left[\bar{\Omega}_{0}^{2} \Delta q^{\prime \prime}+\left(\omega_{n}^{2}-\varepsilon \gamma_{3} F_{1} \cos \bar{\tau}\right) \Delta q+3 \varepsilon \gamma_{4} q_{0}^{2} \Delta q\right] d \bar{\tau}+\frac{1}{T} \int_{0}^{T}(\delta \Delta q)\left[\varepsilon \gamma_{12} \bar{\Omega}_{0}^{\alpha} D_{\bar{\tau}}^{\alpha}(\Delta q)\right] d \bar{\tau} \\
=\frac{1}{2 \pi} \int_{0}^{2 \pi}(\delta \Delta q)\left[-\bar{\Omega}_{0}^{2} q_{0}^{\prime \prime}-\left(\omega_{n}^{2}-\varepsilon \gamma_{3} F_{1} \cos \bar{\tau}\right) q_{0}-\varepsilon \gamma_{4} q_{0}^{3}+\tilde{f} \cos \bar{\tau}\right] d \bar{\tau} \\
-\frac{1}{T} \int_{0}^{T}(\delta \Delta q)\left[\varepsilon \gamma_{12} \bar{\Omega}_{0}^{\alpha} D_{\bar{\tau}}^{\alpha}\left(q_{0}\right)\right] d \bar{\tau}-\frac{1}{2 \pi} \int_{0}^{2 \pi}(\delta \Delta q)\left[2 \bar{\Omega}_{0}^{2} q_{0}^{\prime \prime}\right] d \bar{\tau} \Delta \bar{\Omega} \\
-\frac{1}{T} \int_{0}^{T}(\delta \Delta q)\left[\varepsilon \gamma_{12} \alpha \bar{\Omega}_{0}^{\alpha-1} D_{\bar{\tau}}^{\alpha}\left(q_{0}\right)\right] d \bar{\tau}
\end{gathered}
$$

This yields a system of linearized algebraic equations in terms of $\Delta \boldsymbol{A}$ as

$$
\boldsymbol{M} \Delta \boldsymbol{A}+\boldsymbol{V} \Delta \Omega=\boldsymbol{R}
$$

where elements of the Jacobi matrix $\boldsymbol{M}=\boldsymbol{M}_{1}+\boldsymbol{M}_{2}^{\alpha}$, the corrective vector $\boldsymbol{R}=\boldsymbol{R}_{1}+\boldsymbol{R}_{2}^{\alpha}$ and vector $\boldsymbol{V}=\boldsymbol{V}_{1}+$ $\boldsymbol{V}_{2}^{\alpha}$ are defined as

$$
\begin{gathered}
\boldsymbol{M}_{1}=\frac{1}{2 \pi} \int_{0}^{2 \pi}\left[\bar{\Omega}_{0}^{2} \boldsymbol{C}^{T} \frac{d^{2} \boldsymbol{C}}{d \bar{\tau}^{2}}+\left(\omega_{n}^{2}-\varepsilon \gamma_{3} F_{1} \cos \bar{\tau}\right) \boldsymbol{C}^{T} \boldsymbol{C}+3 \varepsilon \gamma_{4} q_{0}^{2} \boldsymbol{C}^{\boldsymbol{T}} \boldsymbol{C}\right] d \bar{\tau} \\
\boldsymbol{M}_{2}^{\alpha}=-\frac{1}{T} \int_{0}^{T} \boldsymbol{C}^{T}\left[\varepsilon \gamma_{12} \bar{\Omega}_{0}^{\alpha} D_{\bar{\tau}}^{\alpha}(\boldsymbol{C})\right] d \bar{\tau} \\
\boldsymbol{R}_{1}=-\frac{1}{2 \pi} \int_{0}^{2 \pi}\left[\bar{\Omega}_{0}^{2} \boldsymbol{C}^{T} \frac{d^{2} \boldsymbol{C}}{d \bar{\tau}^{2}}+\left(\omega_{n}^{2}-\varepsilon \gamma_{3} F_{1} \cos \bar{\tau}\right) \boldsymbol{C}^{T} \boldsymbol{C}+\varepsilon \gamma_{4} q_{0}^{2} \boldsymbol{C}^{T} \boldsymbol{C}\right] \boldsymbol{A}_{\mathbf{0}}-\tilde{f} \cos \bar{\tau} \boldsymbol{C}^{T} d \bar{\tau} \\
\boldsymbol{R}_{2}^{\alpha}=-\frac{1}{T} \int_{0}^{T} \boldsymbol{C}^{T}\left[\varepsilon \gamma_{12} \bar{\Omega}_{0}^{\alpha} D_{\bar{\tau}}^{\alpha}(\boldsymbol{C})\right] d \bar{\tau} \boldsymbol{A}_{\mathbf{0}} \\
\boldsymbol{V}_{1}=\frac{1}{2 \pi} \int_{0}^{2 \pi}\left[2 \bar{\Omega}_{0}^{2} \boldsymbol{C}^{T} \frac{d^{2} \boldsymbol{C}}{d \bar{\tau}^{2}}\right] d \bar{\tau} \boldsymbol{A}_{\mathbf{0}} \\
\boldsymbol{V}_{2}^{\alpha}=\frac{1}{T} \int_{0}^{T} \boldsymbol{C}^{T}\left[\varepsilon \gamma_{12} \alpha \bar{\Omega}_{0}^{\alpha-1} D_{\bar{\tau}}^{\alpha}(\boldsymbol{C})\right] d \bar{\tau} \boldsymbol{A}_{\mathbf{0}}
\end{gathered}
$$

It should be noted that $\boldsymbol{M}_{2}^{\alpha}$ and $\boldsymbol{R}_{2}^{\alpha}$ are the Jacobi matrix and corrective vector terms corresponding to the fractional-order terms generated by the IHB method. Similar to [35], we can write $\boldsymbol{M}_{2}^{\alpha}, \boldsymbol{R}_{2}^{\alpha}$ and $\boldsymbol{V}_{2}^{\alpha}$ as

$$
\boldsymbol{M}_{2}^{\alpha}=\left[\begin{array}{ll}
{\left[M_{11}\right]^{\alpha}} & {\left[M_{12}\right]^{\alpha}} \\
{\left[M_{21}\right]^{\alpha}} & {\left[M_{22}\right]^{\alpha}}
\end{array}\right], \quad \boldsymbol{R}_{2}^{\alpha}=\left[\begin{array}{c}
R_{10}^{\alpha} \\
R_{1 i}^{\alpha} \\
R_{2 i}^{\alpha}
\end{array}\right], \quad \boldsymbol{V}_{2}^{\alpha}=\left[\begin{array}{c}
V_{10}^{\alpha} \\
V_{1 i}^{\alpha} \\
V_{2 i}^{\alpha}
\end{array}\right], \quad i=1,2, \ldots N .
$$

Derivation of the elements of the matrix $\boldsymbol{M}_{2}^{\alpha}, \boldsymbol{R}_{2}^{\alpha}$ and $\boldsymbol{V}_{2}^{\alpha}$ are given in Appendix A, while more details on this procedure can be also seen in [36]. Finally, fractional-order part of the elements of Eq. (67) are expressed as

$$
\begin{gathered}
{\left[M_{11}\right]_{i j}^{\alpha}=\delta_{i j} \varepsilon \gamma_{12} \bar{\Omega}^{\alpha} \frac{i^{\alpha}}{2} \cos \left(\frac{\alpha \pi}{2}\right), i=0,1,2, \ldots N, j=0,1,2, \ldots N,} \\
{\left[M_{12}\right]_{i j}^{\alpha}=\delta_{i j} \varepsilon \gamma_{12} \bar{\Omega}^{\alpha} \frac{i^{\alpha}}{2} \sin \left(\frac{\alpha \pi}{2}\right), i=0,1,2, \ldots N, j=1,2, \ldots N,} \\
{\left[M_{21}\right]_{i j}^{\alpha}=-\delta_{i j} \varepsilon \gamma_{12} \bar{\Omega}^{\alpha} \frac{i^{\alpha}}{2} \sin \left(\frac{\alpha \pi}{2}\right), i=1,2, \ldots N, j=0,1,2, \ldots N,} \\
{\left[M_{22}\right]_{i j}^{\alpha}=\delta_{i j} \varepsilon \gamma_{12} \bar{\Omega}^{\alpha} \frac{i^{\alpha}}{2} \cos \left(\frac{\alpha \pi}{2}\right), i=1,2, \ldots N, j=1,2, \ldots N,} \\
R_{10}^{\alpha}=0, \\
R_{1 i}^{\alpha}=-\varepsilon \gamma_{12} \bar{\Omega}^{\alpha}\left[a_{i} \frac{i^{\alpha}}{2} \cos \left(\frac{\alpha \pi}{2}\right)+b_{i} \frac{i^{\alpha}}{2} \sin \left(\frac{\alpha \pi}{2}\right)\right], \quad i=1,2, \ldots N,
\end{gathered}
$$




$$
\begin{gathered}
R_{2 i}^{\alpha}=-\varepsilon \gamma_{12} \bar{\Omega}^{\alpha}\left[a_{i} \frac{i^{\alpha}}{2} \sin \left(\frac{\alpha \pi}{2}\right)+b_{i} \frac{i^{\alpha}}{2} \cos \left(\frac{\alpha \pi}{2}\right)\right], \quad i=1,2, \ldots N, \\
V_{10}^{\alpha}=0, \\
V_{1 i}^{\alpha}=\varepsilon \gamma_{12} \alpha \bar{\Omega}_{0}^{\alpha-1}\left[a_{i} \frac{i^{\alpha}}{2} \cos \left(\frac{\alpha \pi}{2}\right)+b_{i} \frac{i^{\alpha}}{2} \sin \left(\frac{\alpha \pi}{2}\right)\right], \quad i=1,2, \ldots N, \\
V_{2 i}^{\alpha}=\varepsilon \gamma_{12} \alpha \bar{\Omega}_{0}^{\alpha-1}\left[a_{i} \frac{i^{\alpha}}{2} \sin \left(\frac{\alpha \pi}{2}\right)+b_{i} \frac{i^{\alpha}}{2} \cos \left(\frac{\alpha \pi}{2}\right)\right], \quad i=1,2, \ldots N,
\end{gathered}
$$

where $\delta_{i j}$ is the Kronecker delta.

It should be noted that for the single frequency solution we take that $\Delta \bar{\Omega}=0$ in Eq. (64). To start the solution process via the Newton-Rapson technique one needs to assume the initial guess of the coefficients $\boldsymbol{A}$ to find the solution for $\boldsymbol{\Delta} \boldsymbol{A}$. This solution is then added to the current estimate of $\boldsymbol{A}$ to obtain a new estimate. This procedure is repeated until the prescribed value of the norm of $\boldsymbol{R}$ is satisfied (e.g. some small value of the order of $10^{-5}$ ). To obtain the frequency response of the system one should increment the value of frequency $\bar{\Omega}$, where the solution at the previous frequency is used as the initial guess to find the solution at the current frequency. For smaller increments of the frequency, one should expect faster convergence at a given frequency.

\section{Numerical study}

Here, we will give the numerical results obtained by the presented methodologies for finding the solution of the fractional-order forced Mathieu-Duffing equation. First, we will validate the results by comparing the steady-state frequency responses for the superharmonic resonance case $2 \bar{\Omega} \approx \omega_{n}$ obtained by two different approaches, the multiple scales, and the incremental harmonic balance method, as given in Fig. 2 a). It should be noted that for the IHB method, $N=8$ terms in Fourier series are used for all numerical examples. The amplitudes for the IHB method corresponding to particular Fourier coefficients and harmonics are calculated as $A_{j}=\sqrt{a_{j}+b_{j}}, j=1,2, \ldots, N$. For this purpose, we observed only the first vibration mode $n=1$ of the simply supported nanobeam and the following values of parameters for the simulations are adopted: $\alpha=0.5, \delta=0.7, G_{p}=0.02, F_{0}=10, F_{1}=6, F_{2}=10, \tilde{R}=1$, and small time scale parameter $\varepsilon=0.1$. For the IHB method, amplitude $A_{2}$ corresponding to the second harmonic is plotted in Fig. 2 a). against the amplitude from multiple scales method for different values of the nonlocal parameter and by using the Eq. (32) and formula $2 \bar{\Omega}=\omega_{n}+\varepsilon \sigma$. A good agreement between the results from the MS and IHB method can be observed for both, upper and lower branches of the amplitude-frequency curves. One can notice a frequency shift in the superharmonic amplitude-frequency curves for an increase of nonlocal parameter i.e. the natural frequency, and consequently the superharmonic resonant frequency decreases for an increase of the nonlocal parameter as expected. However, in the case without or for lower values of the nonlocal parameter, amplitudes from the multiple scales method are higher than those from the IHB method since they decrease for an increase of the nonlocal parameter. This difference could be attributed either to the low-order (first-order) approximation in the MS method or to the application of the IHB without the numerical continuation method, which is unable to calculate unstable branch periodic solutions and bifurcation points. 
Further, verification of the results from the semi-numerical IHB method is performed by comparing them against the results obtained by the numerical technique based on the Newmark method. Fig. 2 b) shows a single periodic solution for the nanobeam system which is compared against the solution obtained by the Grunvald-Letnikov approximation of fractional derivative and procedure based on the Newmark method from the paper by Evangelatos and Spanos [35]. The same values of parameters as in the previous figure are adopted for the simply supported nanobeam with excitation frequency $\bar{\Omega}=1$ and fractional parameter $\alpha=0.5$. We can observe a good agreement between phase curves obtained by the IHB method and the Newmark method.

a)

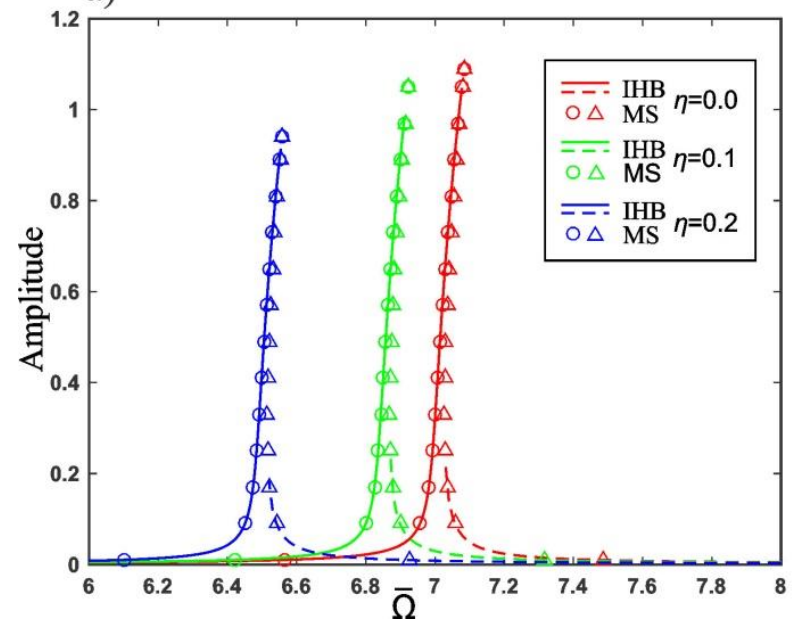

b)

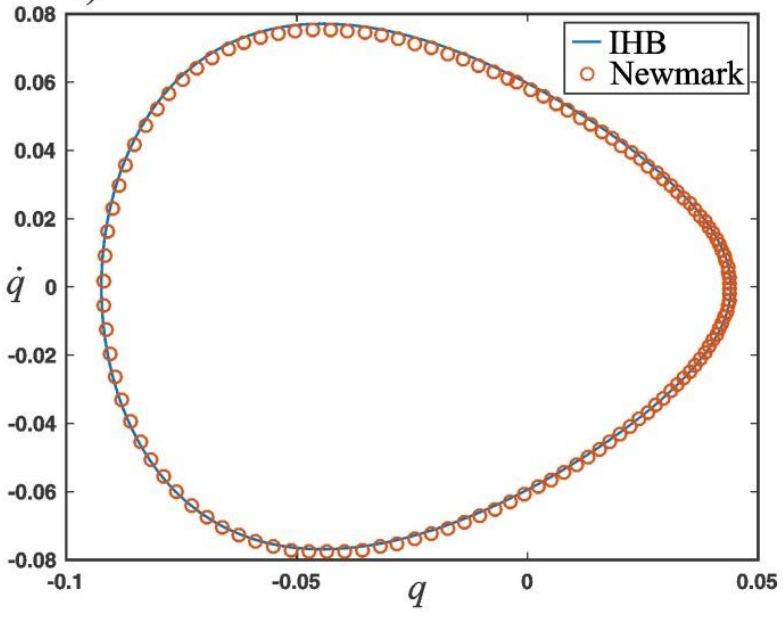

Fig. 2 Verification of the results comparing the a) frequency response curves of the multiple scales and incremental harmonic balance methods (IHBM) for the resonance case $2 \bar{\Omega} \approx \omega_{n}$ and b) a single periodic solution for $\bar{\Omega}=1$ and $\alpha=0.5$ obtained by the IHBM and Newmark method adapted from [35].

\subsection{A parametric study using the multiple scales method}

Here, the parametric study will be performed for the weakly nonlinear case using the multiple time scales method. The multiple scales analysis is performed for the same values of parameters as in the validation study, if not given differently in figures. Figs. 3 and 4 are showing the effect of the dimensionless nonlocal parameter, fractional-order derivative and damping parameter $\delta$ of visco-Pasternak foundation as well as the amplitude of external transverse excitation load on the amplitude-frequency curves. Both simply supported (S-S) and clamped-clamped (C-C) nanobeams are observed for the superharmonic resonance case $2 \bar{\Omega} \approx \omega_{n}$, based on Eq. (41). One can notice that an increase in the fractional-order and damping parameter $\delta$ of the visco-Pasternak foundation leads to a decrease in amplitudes since stronger damping in the system occurs since the order of derivative approaches the first-order derivative i.e. model with pure viscous behavior. Here, we can notice that for the weakly nonlinear case an increase of the nonlocal parameter $\eta$ decreases the amplitudes and slightly bends the amplitude curves to the right (nonlinear hardening effect). This decrease of amplitude due to an increase of the nonlocal parameter is attributed to the softening effect of the nonlocal parameter, which is also confirmed in the literature [29]. The frequency shift that is visible in the validation study cannot be seen here since the lover axis represents the detuning parameter in the vicinity of the resonant frequency and not the excitation frequency itself. For the excitation 
amplitude $F_{2}$ we can notice a decrease in the amplitude-frequency curves for a decrease of the excitation amplitude. From the physical point of view, this means that the clamped nanobeam system is stiffer having a higher natural frequency and lower vibration amplitudes.

A similar tendency can be observed for the amplitude-frequency curves in the case of superharmonic resonance case $3 \bar{\Omega} \approx \omega_{n}$ in Figs. 5 and 6 . The main difference is that amplitudes are generally lower than for the previous superharmonic case. It is a well-known fact that fractional-order derivative damping models exhibit a behaviour that is in between the pure elastic or viscous behaviour depending on the order of derivative. An increase in the fractional-order and damping parameter of the visco-Pasternak foundation leads to a decrease in amplitudes due to the stronger effect of the damping term in the equation. The opposite case occurs for an increase in the amplitude of the excitation load where the amplitude-frequency curves are increasing. The effect of the nonlocal parameter shows similar behavior as for the previous resonant case.

a)

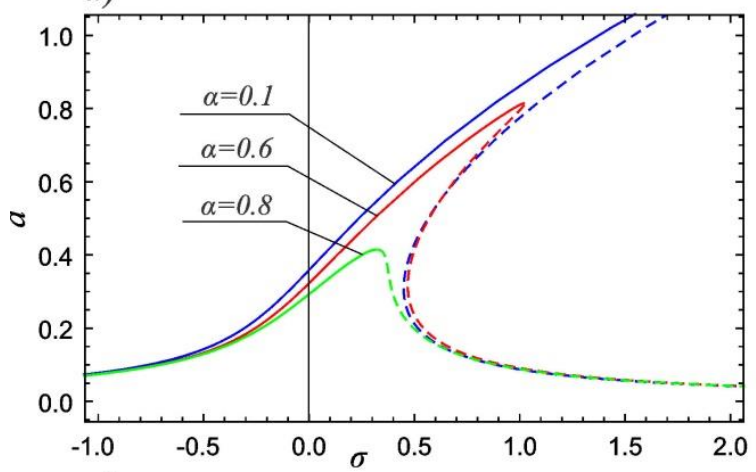

c)

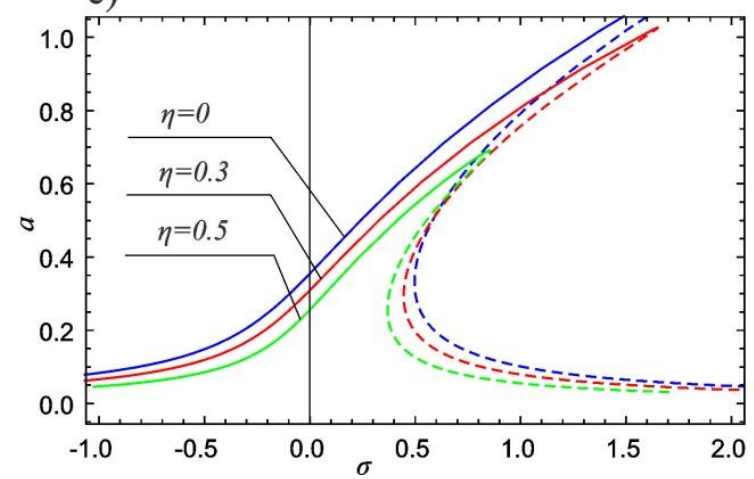

b)

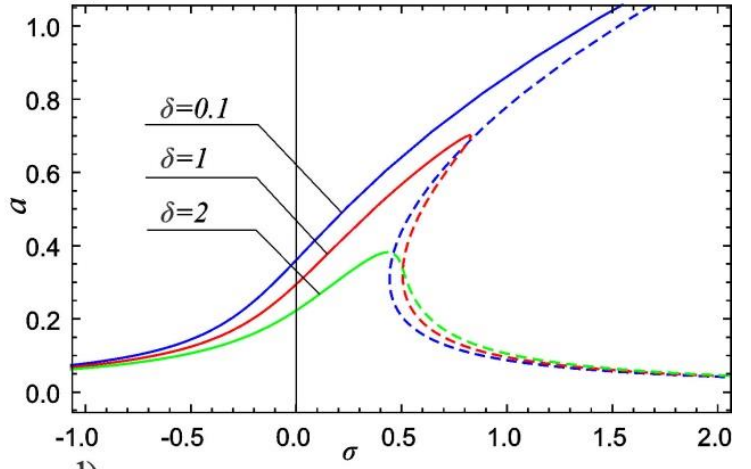

d)

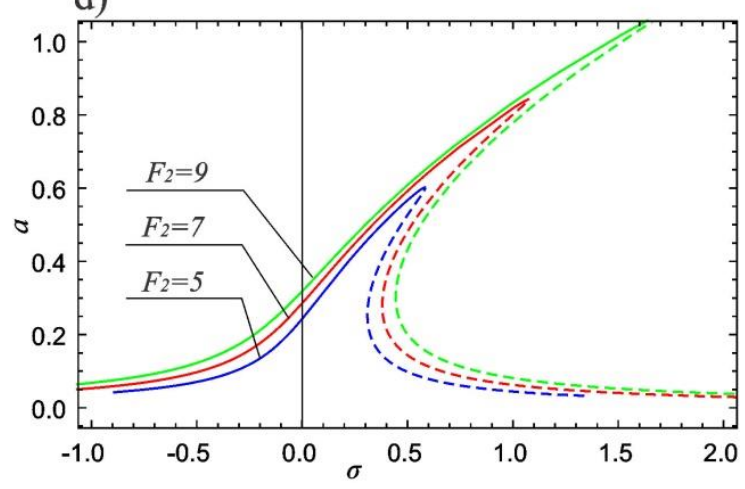

Fig. 3 The frequency response of the S-S nanobeam system for $2 \bar{\Omega} \approx \omega_{n}$. 
a)

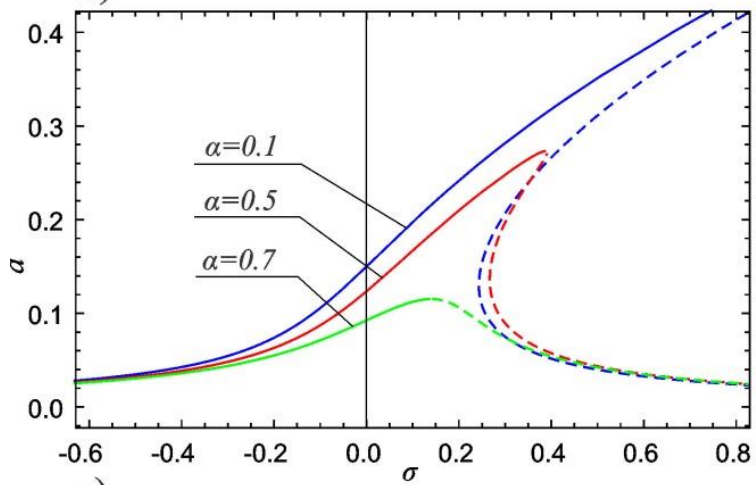

c)

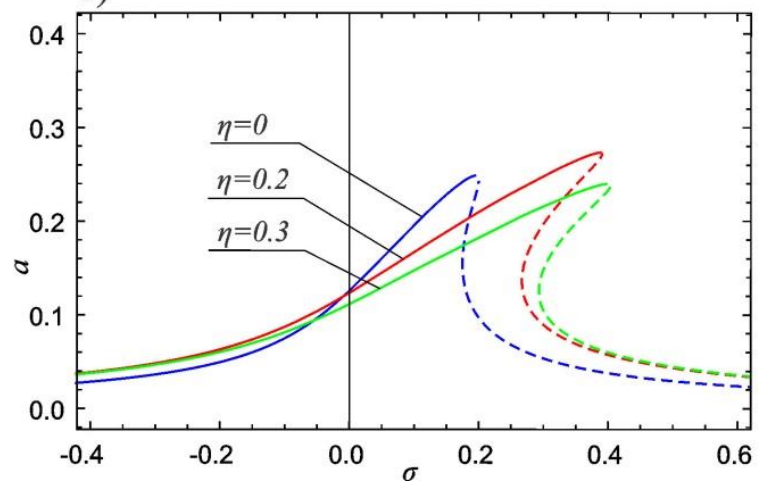

b)

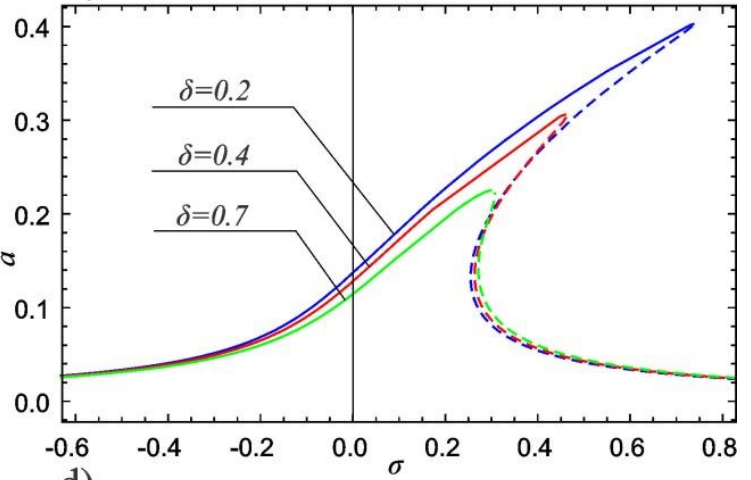

d)

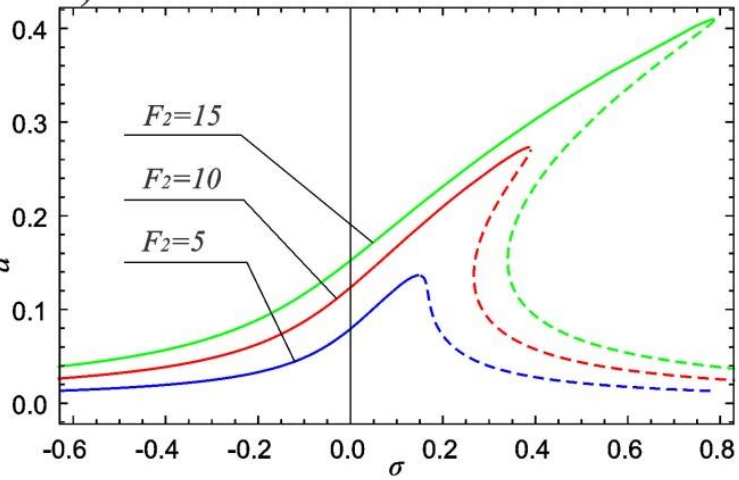

Fig. 4 Frequency response of the C-C nanobeam system for $2 \bar{\Omega} \approx \omega_{n}$.

a)

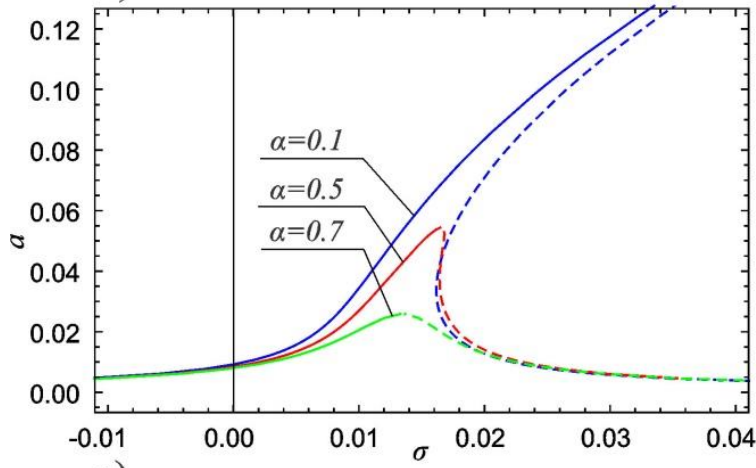

c)

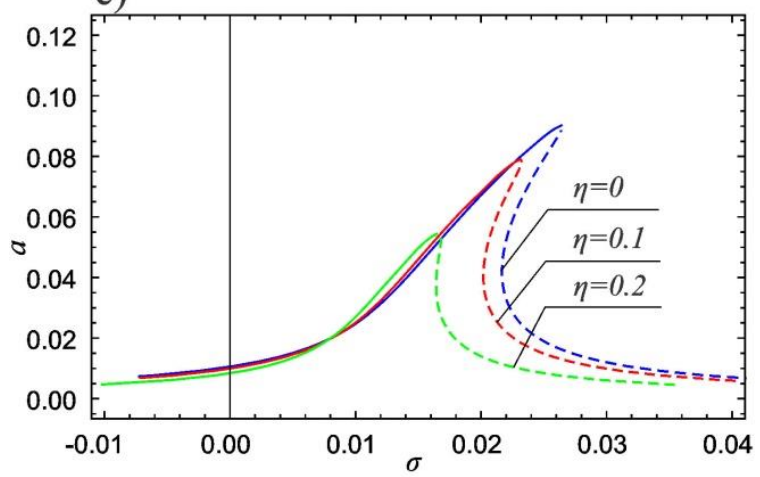

b)

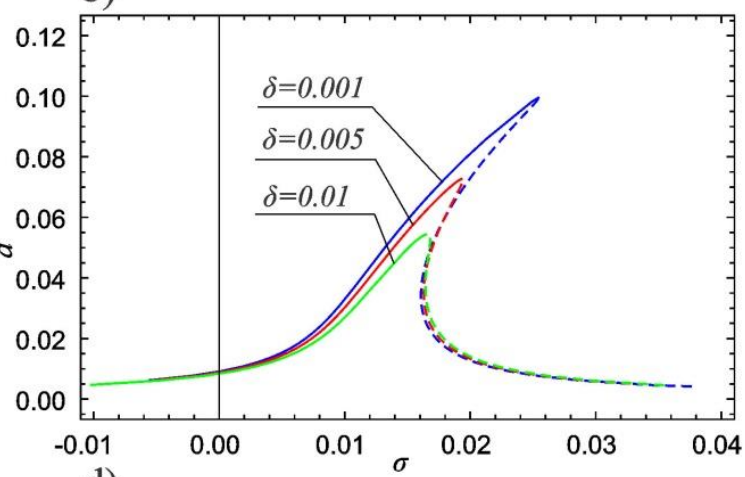

d)

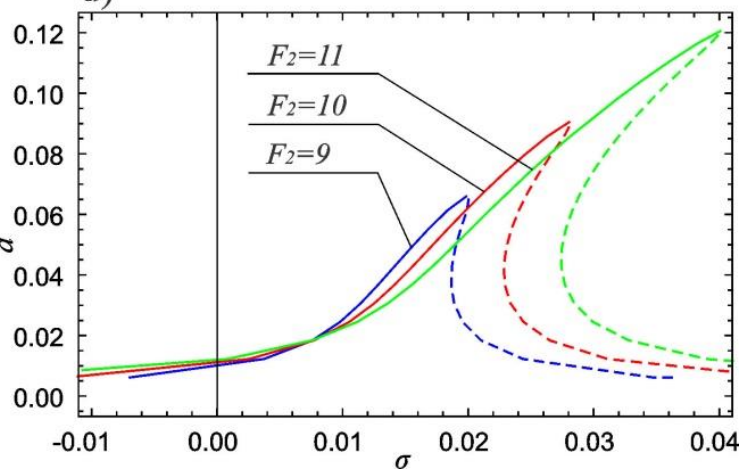

Fig. 5 Frequency response of the S-S nanobeam system for $3 \bar{\Omega} \approx \omega_{n}$. 
a)

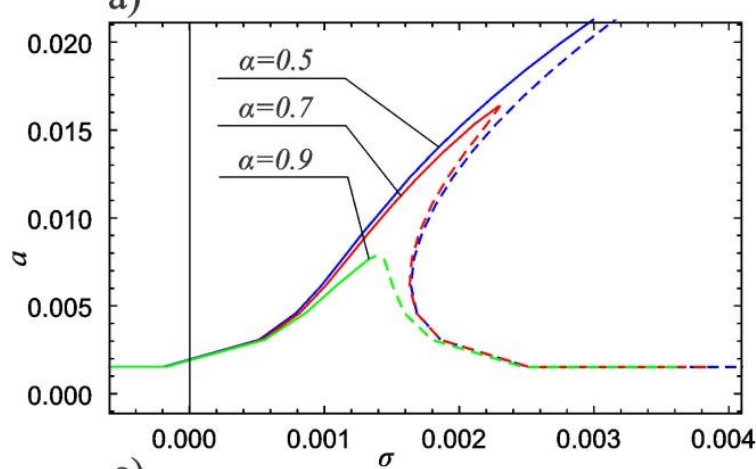

c)

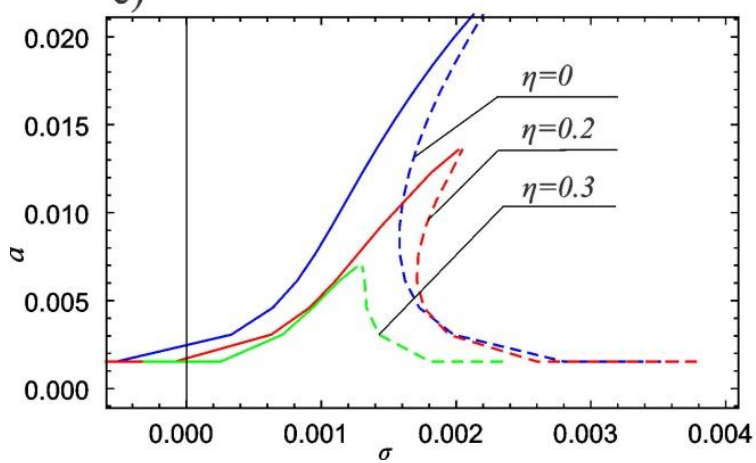

b)

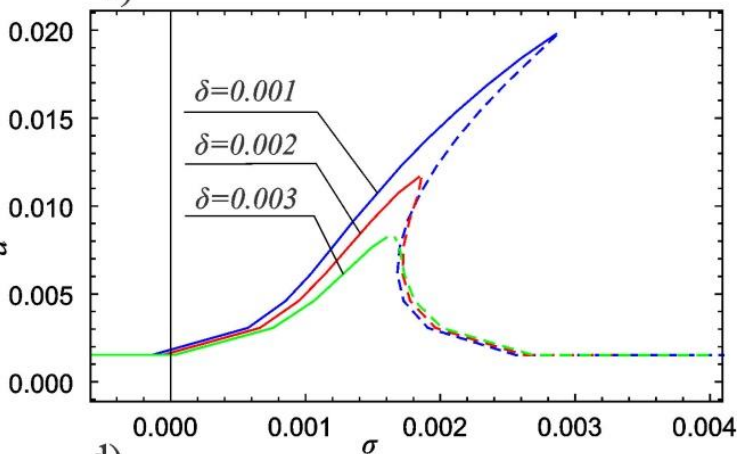

d)

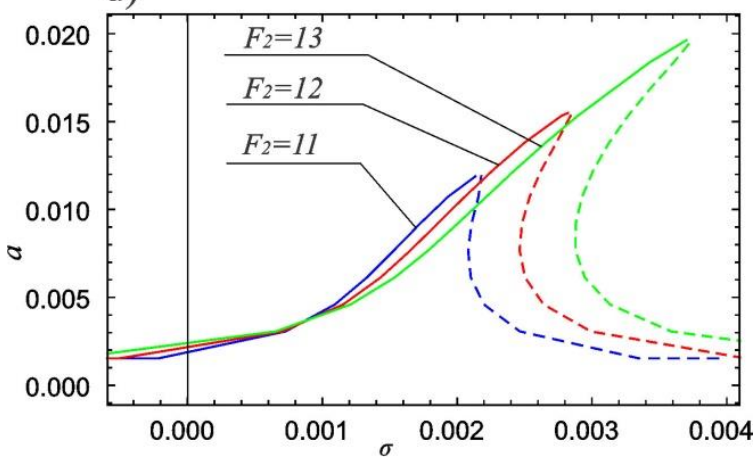

Fig. 6 Frequency response of the C-C nanobeam system for $3 \bar{\Omega} \approx \omega_{n}$.

\subsection{A parametric study using the incremental harmonic method}

As already mentioned, the main reason for including the IHB method when solving the nonlinear differential equation lies in the possibility to study strongly nonlinear oscillations of the system. Here, in Figs. 7 and 8 simulations are performed for the same values of parameters as in the validation study, where the steady-state periodic solutions in the vicinity of the resonance states are again calculated for the weakly nonlinear case $\varepsilon=0.1$. 

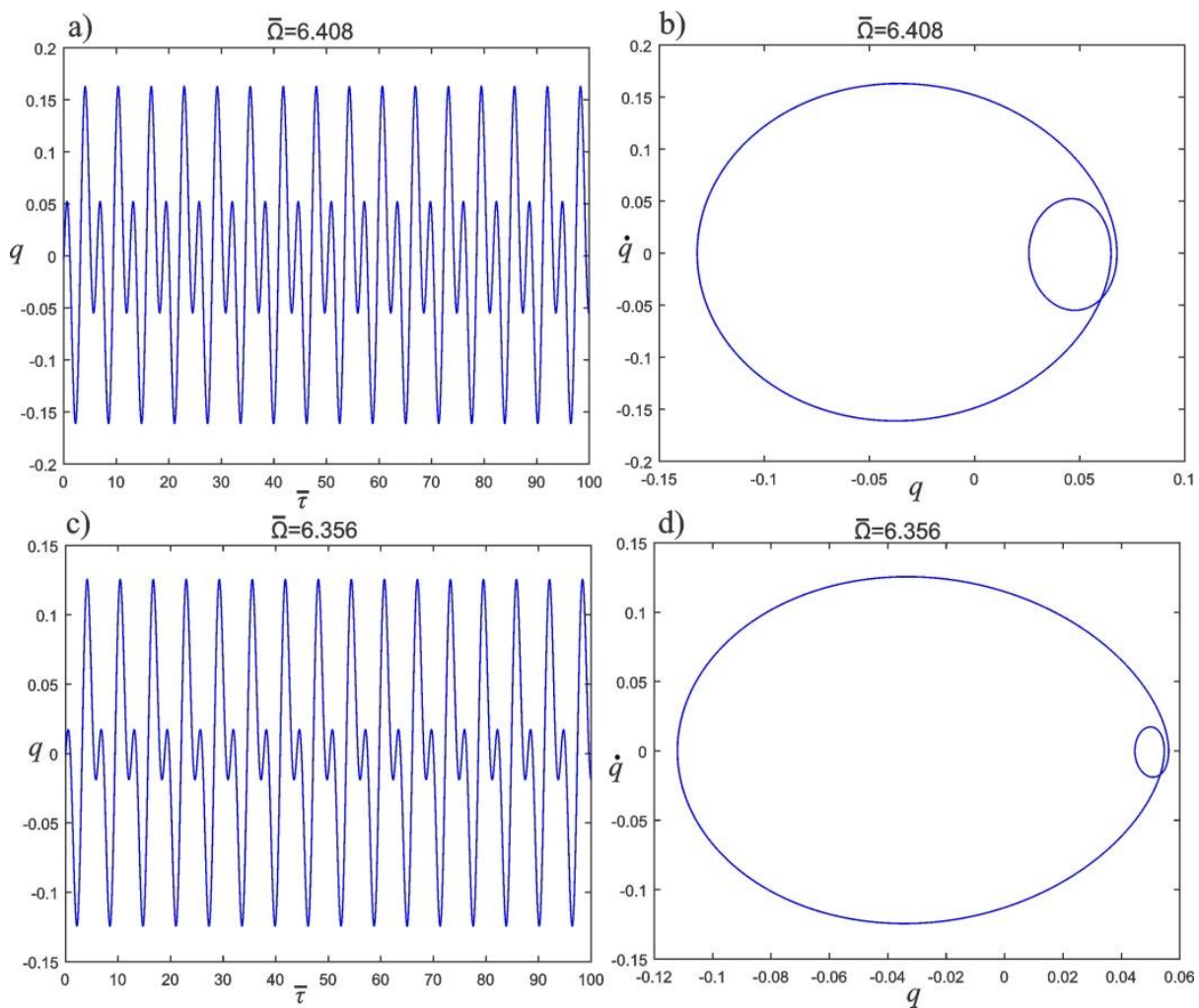

Fig. 7 Time history (left) and phase plane (right) of S-S nanobeam system for the superharmonic resonance $2 \bar{\Omega} \approx \omega_{n}$.
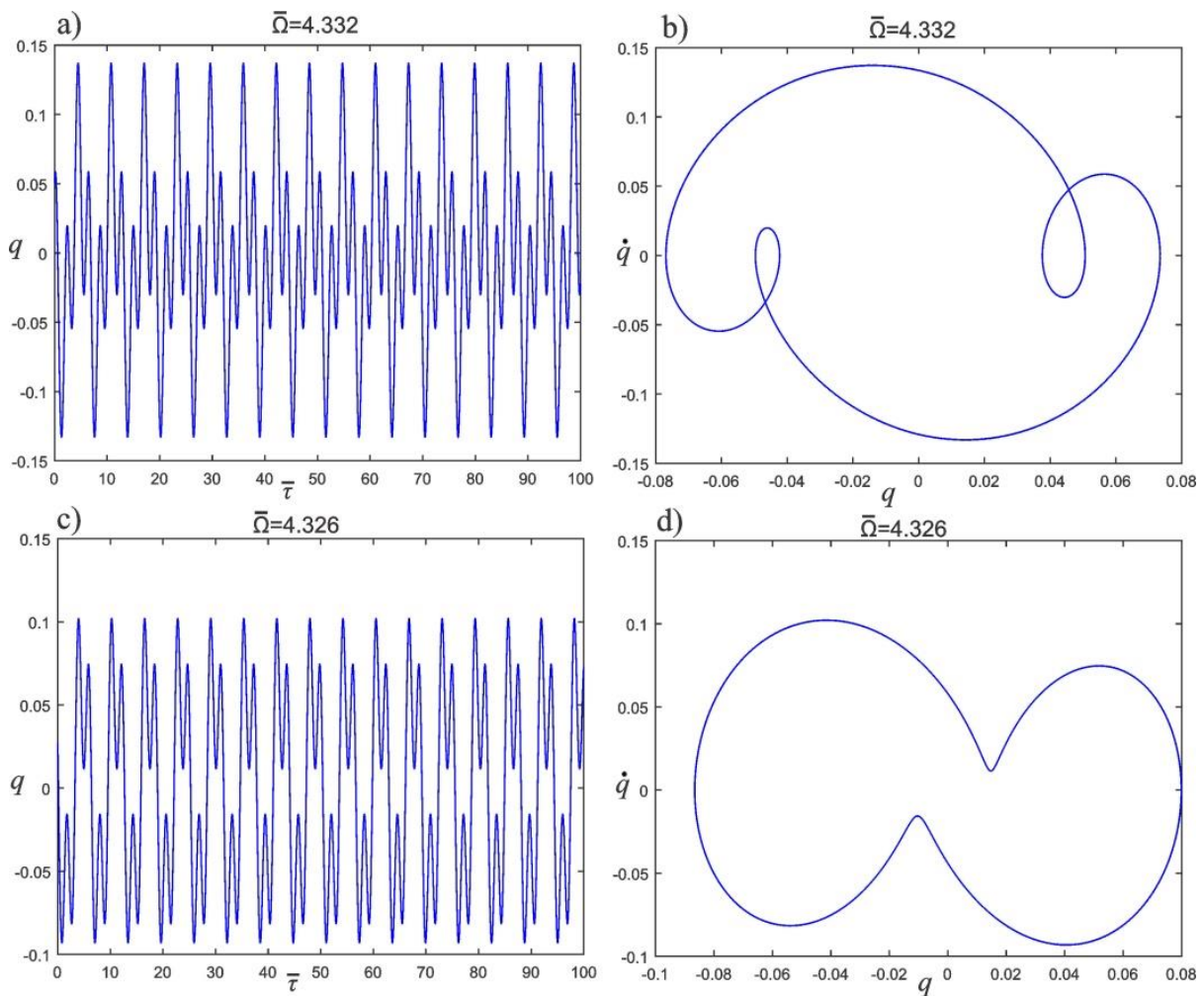

Fig. 8 Time history (left) and phase plane (right) of S-S nanobeam system for the superharmonic resonance $3 \bar{\Omega} \approx \omega_{n}$. 
Figs. 7 and 8 show periodic solutions for the simply supported nanobeam and the superharmonic resonance cases $2 \bar{\Omega} \approx \omega_{n}$ and $3 \bar{\Omega} \approx \omega_{n}$, respectively. In the time history and phase plane, one can notice transformation from regular periodic orbits to the superharmonic one, i.e. one can notice that the system occurs superharmonic resonance, superposition of the first and second harmonic, resulting in the periodic response curves with single loops in the phase plane. Similarly, in Fig. 8 one can observe superposition with the third harmonic which results in two loops in the phase plane curve. This means that in both superharmonic resonance cases the components of the second i.e. third harmonic occupy a dominant position in the amplitudes.

In the following figures, we study the strongly nonlinear case $\varepsilon=1$ by using the incremental harmonic balance method, where we omitted the analysis for the clamped boundary conditions since only reduced amplitudes are obtained without significant qualitative changes. Figs. 9 and 10 show the amplitudefrequency curves of the simply supported nanobeam for changes of the fractional and damping parameters of the visco-Pasternak foundation, nonlocal parameter, and amplitude of external excitation. Since the strongly nonlinear case is observed, amplitudes are larger than those for the weakly nonlinear case obtained by the multiple scales analysis. The effect of the fractional-order damping on amplitudes shows a similar tendency as for the weakly nonlinear case. However, bearing in mind that the assumption of weak damping no longer holds for this case, since there is no influence of the small parameter $\varepsilon$, the effect od fractional damping is stronger. This leads to a significant decrease in amplitudes due to an increase of an order of fractional derivative $\alpha$ or damping parameter $\delta$. However, an increase of the amplitude of external excitation load results in increased amplitudes in the frequency response diagrams. For the nonlocal parameter, we can notice a difference in the behavior for the strongly nonlinear case compared to the weakly nonlinear case analysed via multiple scales method as discussed earlier. For both superharmonic cases, one can observe a significant shift of frequency curves to the left for an increase of the nonlocal parameter. From the literature, it is well known that the introduction of the nonlocal parameter decreases the natural frequency of the system and, therefore, it causes shifting of the superharmonic resonance curves in the amplitude-frequency diagram. Analysis of strongly nonlinear systems via the IHB method can lead to different results and conclusions and possibly provide a more reliable analysis of nanostructures based on nonlocal models. However, further investigation of this problem is necessary. 

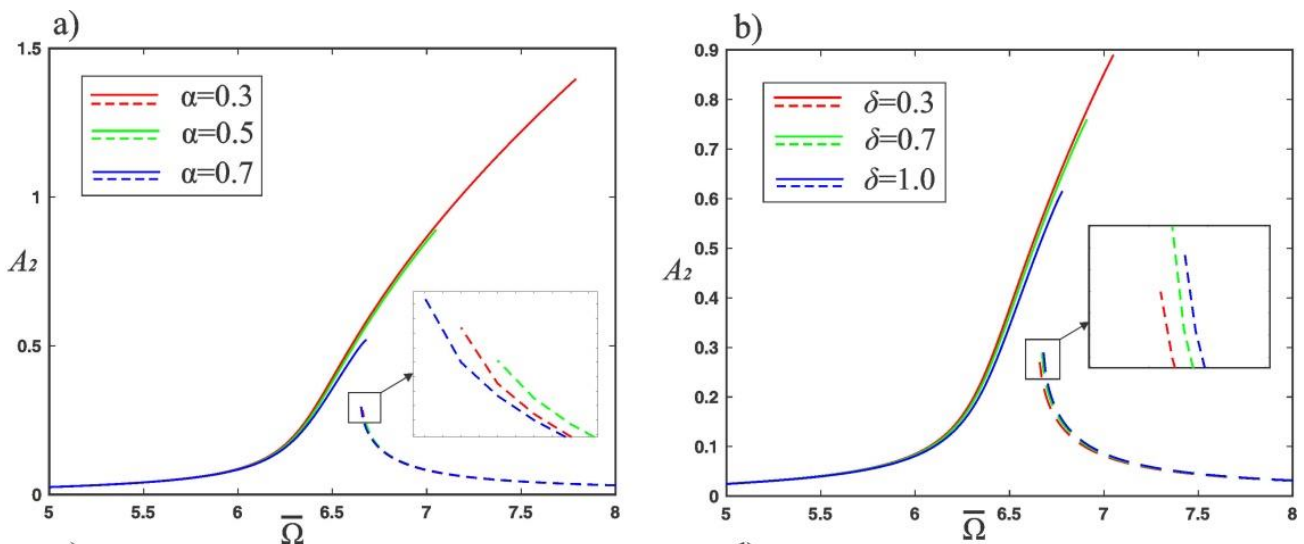

c)
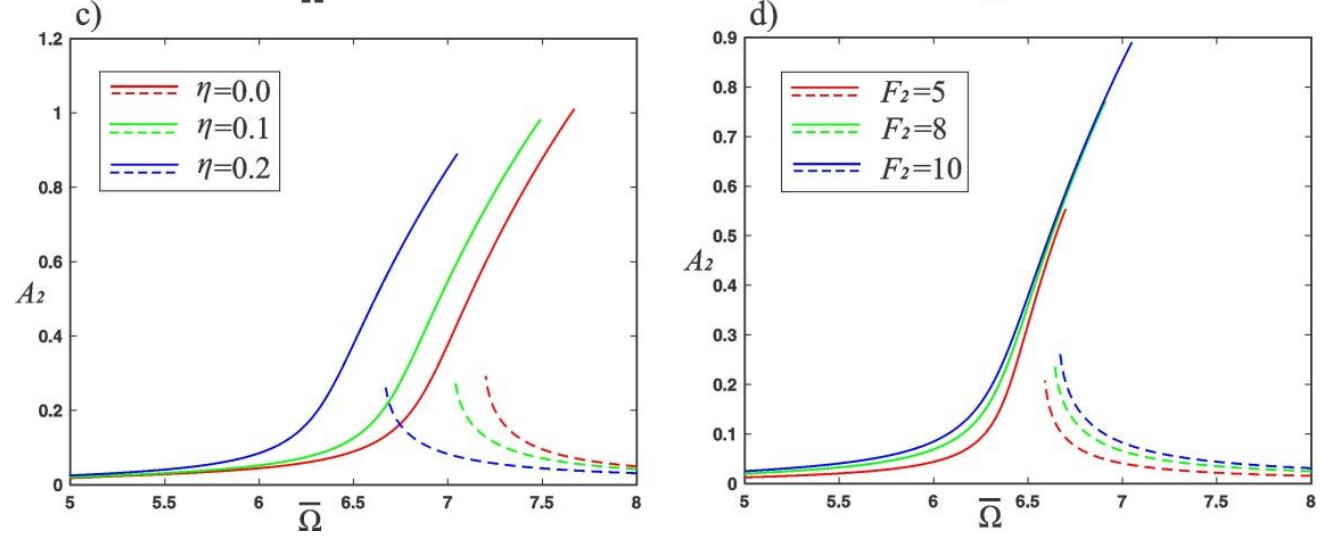

Fig. 9 Amplitude-frequency response of S-S nanobeam for a strongly nonlinear case and the superharmonic resonance $2 \bar{\Omega} \approx \omega_{n}$.
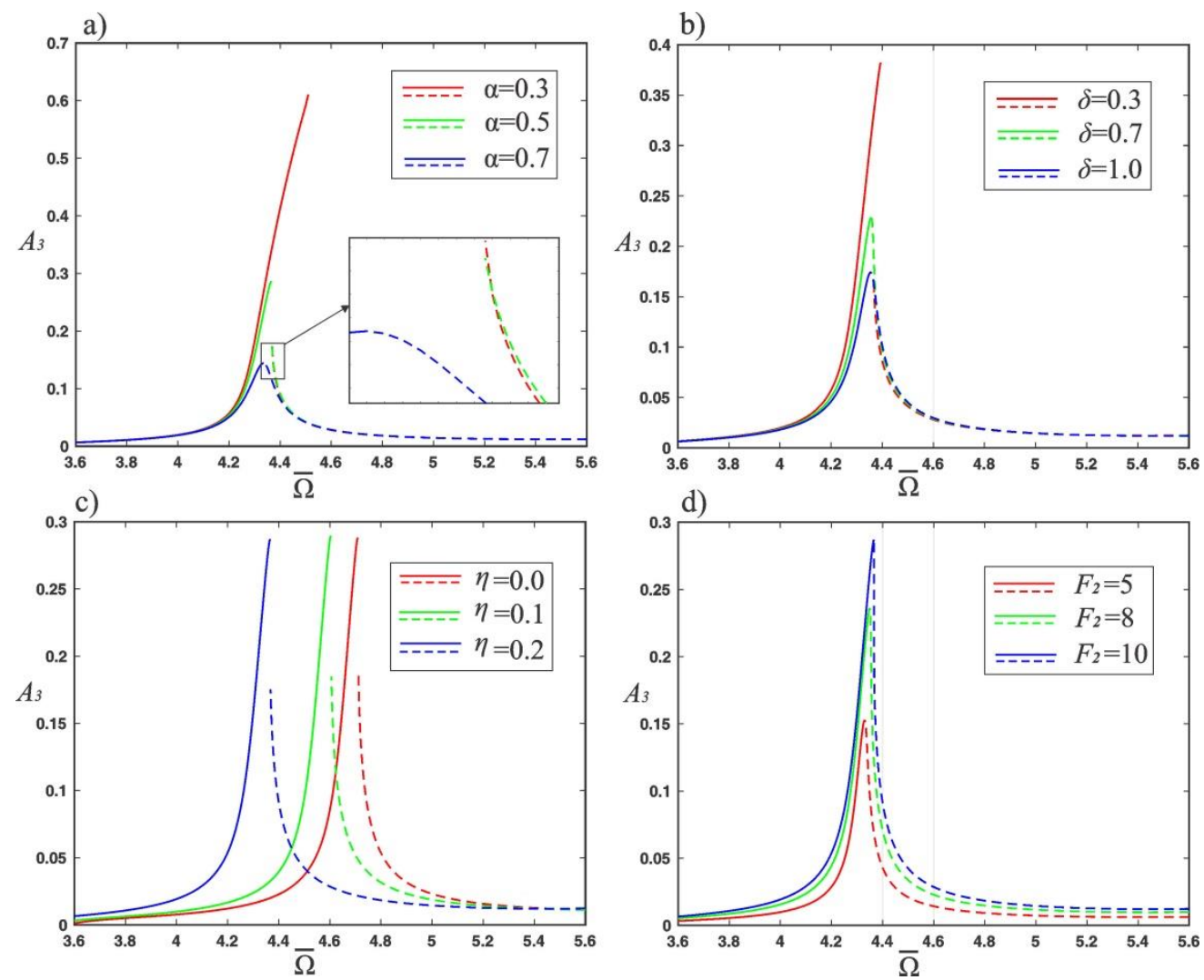

Fig. 10 Amplitude-frequency response of S-S nanobeam for a strongly nonlinear case and the superharmonic resonance $3 \bar{\Omega} \approx \omega_{n}$. 


\section{Conclusion}

In this communication, we analysed the nonlinear vibration of a nanobeam resting on the fractional visco-Pasternak foundation using the nonlocal elasticity theory and fractional-order rheological models. The governing equation is discretized by using the Galerkin approximation and corresponding nonlinear fractional-order forced Mathieu-Duffing equation is obtained. The solution is sought for the steady-state superharmonic resonance conditions using the multiple time scales analysis for the weakly nonlinear case and incremental harmonic balance method for the strongly nonlinear case. The main contributions and conclusions are:

- One-mode Galerkin approximation shown to be effective in discretization of the governing equation for nonlinear nanobeam resting on fractional visco-Pasternak foundation.

- The validation study confirmed the accuracy of the incremental harmonic balance method in obtaining the results for the fractional order nonlinear differential equations.

- The advantage of the incremental harmonic balance method in analysing both weak and strongly nonlinear cases was demonstrated through several numerical examples. However, the obtained amplitude-frequency curves, especially for unstable periodic solutions, have shown deficiency of this method when used without numerical continuation method.

- Parametric study revealed a significant influence of the nonlocal parameter and parameters of the fractional visco-Pasternak foundation on amplitude-frequency response of the system for superharmonic resonance conditions.

This study can be useful for future investigation of the nonlinear behaviour of more complex nanostructure based system with fractional damping by using the combination of the incremental harmonic balance and numerical continuation methods.

Acknowledgments This work was supported by the Serbian Ministry of Education, Science and Technological Development through Mathematical Institute of the Serbian Academy of Sciences and Arts. D.K. was funded by the Marie Skłodowska-Curie Actions - European Commission fellowship (grant number 799201- METACTIVE).

This paper is a contribution in honour of the 75th birthday and 52 years of scientific work of Professor Katica (Stevanović) Hedrih.

\section{References}

[1] Gudmundson P. On the accuracy of the harmonic balance method concerning vibrations of beams with nonlinear supports. Ingenieur-Archiv. 1989; 59(5):333-44.

[2] Sze KY, Chen SA, Huang JL. The incremental harmonic balance method for nonlinear vibration of axially moving beams. Journal of sound and vibration. 2005;281(3-5): 611-26.

[3] Yang XD, Tang YQ, Chen LQ, Lim CW. Dynamic stability of axially accelerating Timoshenko beam: Averaging method. European Journal of Mechanics-A/Solids. 2010; 29(1):81-90. 
[4] Rossikhin YA, Shitikova MV. Free damped nonlinear vibrations of a viscoelastic plate under two-to-one internal resonance. In Materials science forum 2003 (Vol. 440, pp. 29-36). Trans Tech Publications Ltd.

[5] Bucher C, Pirrotta A. Dynamic Finite Element analysis of fractionally damped structural systems in the time domain. Acta Mechanica. 2015; 226(12):3977-90.

[6] Camponeschi E, Vance R, Al-Haik M, Garmestani H, Tannenbaum R. Properties of carbon nanotubepolymer composites aligned in a magnetic field. Carbon. 2007; 45(10):2037-46.

[7] Arani AG, Dashti P, Amir S, Yousefi M. Nonlinear vibration of coupled nano-and microstructures conveying fluid based on Timoshenko beam model under two-dimensional magnetic field. Acta Mechanica. 2015; 226(8):2729-60.

[8] Pavlović IR, Pavlović R, Janevski G, Despenić N, Pajković V. Dynamic behaviour of two elastically connected nanobeams under a white noise process. Facta Universitatis, Series: Mechanical Engineering. 2020.

[9] Mahmoud, F. F., Eltaher, M. A., Alshorbagy, A. E., \& Meletis, E. I. (2012). Static analysis of nanobeams including surface effects by nonlocal finite element. Journal of Mechanical Science and Technology, 26(11), 3555-3563.

[10] Eltaher, M. A., Mahmoud, F. F., Assie, A. E., \& Meletis, E. I. (2013). Coupling effects of nonlocal and surface energy on vibration analysis of nanobeams. Applied Mathematics and Computation, 224, 760-774.

[11] Eltaher, M. A., Khater, M. E., \& Emam, S. A. (2016). A review on nonlocal elastic models for bending, buckling, vibrations, and wave propagation of nanoscale beams. Applied Mathematical Modelling, 40(5-6), 4109-4128.

[12] Eltaher, M. A., Hamed, M. A., Sadoun, A. M., \& Mansour, A. (2014). Mechanical analysis of higher order gradient nanobeams. Applied Mathematics and Computation, 229, 260-272.

[13] Agwa, M. A., \& Eltaher, M. A. (2016). Vibration of a carbyne nanomechanical mass sensor with surface effect. Applied Physics A, 122(4), 335.

[14] Eltaher, M. A., Abdraboh, A. M., \& Almitani, K. H. (2018). Resonance frequencies of size dependent perforated nonlocal nanobeam. Microsystem Technologies, 24(9), 3925-3937.

[15] Eltaher, M. A., Omar, F. A., Abdalla, W. S., \& Gad, E. H. (2019). Bending and vibrational behaviors of piezoelectric nonlocal nanobeam including surface elasticity. Waves in Random and Complex Media, 29(2), 264-280.

[16] Sedighi, H. M., \& Malikan, M. (2020). Stress-driven nonlocal elasticity for nonlinear vibration characteristics of carbon/boron-nitride hetero-nanotube subject to magneto-thermal environment. Physica Scripta, 95(5), 055218.

[17] Sedighi, H. M., Malikan, M., Valipour, A., \& Żur, K. K. (2020). Nonlocal vibration of carbon/boron-nitride nano-hetero-structure in thermal and magnetic fields by means of nonlinear finite element method. Journal of Computational Design and Engineering.

[18] Hamed, M. A., Mohamed, S. A., \& Eltaher, M. A. (2020). Buckling analysis of sandwich beam rested on elastic foundation and subjected to varying axial in-plane loads. Steel and Composite Structures, 34(1), 75-89. 
[19] Mohamed, N., Eltaher, M. A., Mohamed, S. A., \& Seddek, L. F. (2019). Energy equivalent model in analysis of postbuckling of imperfect carbon nanotubes resting on nonlinear elastic foundation. Structural Engineering and Mechanics, 70(6), 737-750.

[20] Mohamed, N., Mohamed, S. A., \& Eltaher, M. A. (2020). Buckling and post-buckling behaviors of higher order carbon nanotubes using energy-equivalent model. Engineering with Computers, 1-14.

[21] Eltaher, M. A., Mohamed, N., Mohamed, S. A., \& Seddek, L. F. (2019). Periodic and nonperiodic modes of postbuckling and nonlinear vibration of beams attached to nonlinear foundations. Applied Mathematical Modelling, 75, 414-445.

[22] Emam, S. A., Eltaher, M. A., Khater, M. E., \& Abdalla, W. S. (2018). Postbuckling and free vibration of multilayer imperfect nanobeams under a pre-stress load. Applied Sciences, 8(11), 2238.

[23] Mohamed, N., Eltaher, M. A., Mohamed, S. A., \& Seddek, L. F. (2018). Numerical analysis of nonlinear free and forced vibrations of buckled curved beams resting on nonlinear elastic foundations. International Journal of Non-Linear Mechanics, 101, 157-173.

[24] Cajić M, Lazarević M, Karličić D, Sun H, Liu X. Fractional-order model for the vibration of a nanobeam influenced by an axial magnetic field and attached nanoparticles. Acta Mechanica. 2018; 229(12):4791815.

[25] Ansari R, Oskouie MF, Sadeghi F, Bazdid-Vahdati M. Free vibration of fractional viscoelastic Timoshenko nanobeams using the nonlocal elasticity theory. Physica E: Low-Dimensional Systems and Nanostructures. 2015; 74:318-27.

[26] Cajić M, Karličić D, Lazarević M. Nonlocal vibration of a fractional order viscoelastic nanobeam with attached nanoparticle. Theoretical and Applied Mechanics. 2015; 42(3):167-90.

[27] Ansari R, Oskouie MF, Gholami R. Size-dependent geometrically nonlinear free vibration analysis of fractional viscoelastic nanobeams based on the nonlocal elasticity theory. Physica E: Low-Dimensional Systems and Nanostructures. 2016; 75:266-71.

[28] Oskouie MF, Ansari R. Linear and nonlinear vibrations of fractional viscoelastic Timoshenko nanobeams considering surface energy effects. Applied Mathematical Modelling. 2017; 43:337-50.

[29] Eyebe GJ, Betchewe G, Mohamadou A, Kofane TC. Nonlinear vibration of a nonlocal nanobeam resting on fractional-order viscoelastic Pasternak foundations. Fractal and Fractional. 2018; 2(3):21.

[30] Farhatnia F, Ghanbari-Mobarakeh M, Rasouli-Jazi S, Oveissi S. Thermal buckling analysis of functionally graded circular plate resting on the pasternak elastic foundation via the differential transform method. Facta Universitatis, Series: Mechanical Engineering. 2017; 15(3):545-63.

[31] Jha AK, Dasgupta SS. Mathematical modeling of a fractionally damped nonlinear nanobeam via nonlocal continuum approach. Proceedings of the Institution of Mechanical Engineers, Part C: Journal of Mechanical Engineering Science. 2019; 233(19-20):7101-15.

[32] Shitikova MV. The fractional derivative expansion method in nonlinear dynamic analysis of structures. Nonlinear Dynamics. 2020; 99(1):109-22.

[33] Niu J, Shen Y, Yang S, Li S. Higher-order approximate steady-state solutions for strongly nonlinear systems by the improved incremental harmonic balance method. Journal of Vibration and Control. $2018 ; 24(16): 3744-57$. 
[34] Shen JH, Lin KC, Chen SH, Sze KY. Bifurcation and route-to-chaos analyses for Mathieu-Duffing oscillator by the incremental harmonic balance method. Nonlinear Dynamics. 2008; 52(4):403-14.

[35] Shen YJ, Wen SF, Li XH, Yang SP, Xing HJ. Dynamical analysis of fractional-order nonlinear oscillator by incremental harmonic balance method. Nonlinear Dynamics. 2016; 85(3):1457-67.

[36] Wen SF, Shen YJ, Wang XN, Yang SP, Xing HJ. Dynamical analysis of strongly nonlinear fractional-order Mathieu-Duffing equation. Chaos: An Interdisciplinary Journal of Nonlinear Science. 2016; 26(8):084309.

[37] Sourani P, Hashemian M, Pirmoradian M, Toghraie D. A Comparison of the Bolotin and Incremental Harmonic Balance Methods in the Dynamic Stability Analysis of an Euler-Bernoulli Nanobeam Based on the Nonlocal Strain Gradient Theory and Surface Effects. Mechanics of Materials. 2020: 103403.

[38] Karličić D, Kozić P, Pavlović R, Nešić N. Dynamic stability of single-walled carbon nanotube embedded in a viscoelastic medium under the influence of the axially harmonic load. Composite Structures. 2017; 162:227-43.

[39] Lewandowski R, Wielentejczyk P. Nonlinear vibration of viscoelastic beams described using fractional order derivatives. Journal of Sound and Vibration. 2017; 399:228-43.

[40] Wielentejczyk P, Lewandowski R. Geometrically nonlinear, steady state vibration of viscoelastic beams. International Journal of Non-Linear Mechanics. 2017; 89:177-86.

[41] Eringen AC, Edelen DG. On nonlocal elasticity. International Journal of Engineering Science. 197; 10(3):233-48.

[42] Eringen AC. On differential equations of nonlocal elasticity and solutions of screw dislocation and surface waves. Journal of applied physics. 1983; 54(9):4703-10.

[43] Atanacković TM, Pilipović S, Stanković B, Zorica D. Fractional calculus with applications in mechanics. Wiley-ISTE; 2014.

[44] Cai W, Chen W, Xu W. Fractional modeling of Pasternak-type viscoelastic foundation. Mechanics of Time-Dependent Materials. 2017 Feb 1;21(1):119-31.

[45] Nayfeh AH. On the Discretization of Spatially Continuous Systems with Quadratic and Cubic Nonlinearities. JSME International Journal, Series C. 2002; 45(1):79-86.

[46] Kovacic I, Brennan MJ. The Duffing equation: nonlinear oscillators and their behaviour. John Wiley \& Sons; 2011.

[47] Podlubny I. Fractional differential equations: an introduction to fractional derivatives, fractional differential equations, to methods of their solution and some of their applications. Elsevier; 1998.

[48] Ansari R, Sahmani S. Small scale effect on vibrational response of single-walled carbon nanotubes with different boundary conditions based on nonlocal beam models. Communications in Nonlinear Science and Numerical Simulation. 2012; 17(4):1965-79.

[49] Ramakrishnan V, Feeny BF. Resonances of a forced Mathieu equation with reference to wind turbine blades. Journal of vibration and acoustics. 2012; 134(6).

[50] Evangelatos GI, Spanos PD. An accelerated newmark scheme for integrating the equation of motion of nonlinear systems comprising restoring elements governed by fractional derivatives. In Recent advances in mechanics 2011 (pp. 159-177). Springer, Dordrecht. 
[51] Noll MU, Lentz L, Wagner UV. On the discretization of a bistable cantilever beam with application to energy harvesting. Facta Universitatis, Series: Mechanical Engineering. 2019; 17(2):125-139.

[52] Yang, W. D., \& Wang, X. (2016). Nonlinear pull-in instability of carbon nanotubes reinforced nanoactuator with thermally corrected Casimir force and surface effect. International Journal of Mechanical Sciences, 107, 34-42.

[53] Sedighi, H. M., \& Farjam, N. (2017). A modified model for dynamic instability of CNT based actuators by considering rippling deformation, tip-charge concentration and Casimir attraction. Microsystem technologies, 23(6), 2175-2191.

\section{Appendix A}

Here, due to simplicity, we will present the procedure of integrating the fractional-order term in Jacobi matrix and corrective vector that is described in detail in the paper by Shen [19] The elements of the matrix $\boldsymbol{M}_{2}^{\alpha}$ and vector $\boldsymbol{R}_{2}^{\alpha}$ from Eq. (71) based on Eqs. (68) and (70) and elements of the matrix $\boldsymbol{V}_{2}^{\alpha}$ Eq. (85) based on Eq. (84) are given as

$$
\begin{gathered}
{\left[M_{11}\right]_{i j}^{\alpha}=\lim _{T \rightarrow \infty} \frac{1}{T} \int_{0}^{T} \varepsilon \gamma_{12} \bar{\Omega}^{\alpha} \cos (i \bar{\tau}) D_{\bar{\tau}}^{\alpha}[\cos (j \bar{\tau})] d \bar{\tau}, \quad i=0,1,2, \ldots N, j=0,1,2, \ldots N,} \\
{\left[M_{12}\right]_{i j}^{\alpha}=\lim _{T \rightarrow \infty} \frac{1}{T} \int_{0}^{T} \varepsilon \gamma_{12} \bar{\Omega}^{\alpha} \cos (i \bar{\tau}) D_{\bar{\tau}}^{\alpha}[\sin (j \bar{\tau})] d \bar{\tau}, \quad i=0,1,2, \ldots N, j=1,2, \ldots N,} \\
{\left[M_{21}\right]_{i j}^{\alpha} \lim _{T \rightarrow \infty} \frac{1}{T} \int_{0}^{T} \varepsilon \gamma_{12} \bar{\Omega}^{\alpha} \sin (i \bar{\tau}) D_{\bar{\tau}}^{\alpha}[\cos (j \bar{\tau})] d \bar{\tau}, \quad i=1,2, \ldots N, j=0,1,2, \ldots N,} \\
{\left[M_{22}\right]_{i j}^{\alpha}=\lim _{T \rightarrow \infty} \frac{1}{T} \int_{0}^{T} \varepsilon \gamma_{12} \bar{\Omega}^{\alpha} \sin (i \bar{\tau}) D_{\bar{\tau}}^{\alpha}[\sin (j \bar{\tau})] d \bar{\tau}, \quad i=1,2, \ldots N, j=1,2, \ldots N .} \\
R_{10}^{\alpha}=\lim _{T \rightarrow \infty}-\frac{1}{T} \int_{0}^{T} \varepsilon \gamma_{12} \bar{\Omega}^{\alpha} D_{\bar{\tau}}^{\alpha}\left[q_{0}\right] d \bar{\tau}, \\
R_{1 i}^{\alpha}=\lim _{T \rightarrow \infty}-\frac{1}{T} \int_{0}^{T} \varepsilon \gamma_{12} \bar{\Omega}^{\alpha} D_{\bar{\tau}}^{\alpha}\left[q_{0}\right] \cos (i \bar{\tau}) d \bar{\tau}, \quad i=1,2, \ldots N, \\
R_{2 i}^{\alpha}=\lim _{T \rightarrow \infty}-\frac{1}{T} \int_{0}^{T} \varepsilon \gamma_{12} \bar{\Omega}^{\alpha} D_{\bar{\tau}}^{\alpha}\left[q_{0}\right] \sin (i \bar{\tau}) d \bar{\tau}, \quad i=1,2, \ldots N, \\
V_{10}^{\alpha}=\lim _{T \rightarrow \infty} \frac{1}{T} \int_{0}^{T} \varepsilon \gamma_{12} \alpha \bar{\Omega}_{0}^{\alpha-1} D_{\bar{\tau}}^{\alpha}\left[q_{0}\right] d \bar{\tau}, \\
V_{1 i}^{\alpha}=\lim _{T \rightarrow \infty} \frac{1}{T} \int_{0}^{T} \varepsilon \gamma_{12} \alpha \bar{\Omega}_{0}^{\alpha-1} D_{\bar{\tau}}^{\alpha}\left[q_{0}\right] \cos (i \bar{\tau}) d \bar{\tau}, \\
i=1,2, \ldots N, \\
i \gamma_{12} \alpha \bar{\Omega}_{0}^{\alpha-1} D_{\bar{\tau}}^{\alpha}\left[q_{0}\right] \sin (i \bar{\tau}) d \bar{\tau}, \quad i=1,2, \ldots N, \\
\end{gathered}
$$

Based on Caputo's definition of fractional derivative we can rewrite Eq. (A1) as

$$
\begin{aligned}
{\left[M_{11}\right]_{i j}^{\alpha} } & =\lim _{T \rightarrow \infty} \frac{1}{T} \int_{0}^{T} \varepsilon \gamma_{12} \bar{\Omega}^{\alpha} \cos (i \bar{\tau}) D_{\bar{\tau}}^{\alpha}[\cos (j \bar{\tau})] d \bar{\tau} \\
& =\lim _{T \rightarrow \infty} \frac{1}{T} \int_{0}^{T} \varepsilon \gamma_{12} \bar{\Omega}^{\alpha} \cos (i \bar{\tau})\left[\frac{1}{\Gamma(1-\alpha)} \int_{0}^{\bar{\tau}} \frac{-j \sin (j r)}{(\bar{\tau}-r)^{\alpha}} d r\right] d \bar{\tau} .
\end{aligned}
$$

If we take that $s=\bar{\tau}-r$ and $d r=-d s$, then Eq. (A11) is given as 


$$
\begin{aligned}
{\left[M_{11}\right]_{i j}^{\alpha}=} & -\frac{j \varepsilon \gamma_{12} \bar{\Omega}^{\alpha}}{\Gamma(1-\alpha)} \lim _{T \rightarrow \infty} \frac{1}{T} \int_{0}^{T} \cos (i \bar{\tau})\left[\int_{0}^{\bar{\tau}} \frac{\sin (j \bar{\tau}-j s)}{s^{\alpha}} d s\right] d \bar{\tau} . \\
& =\frac{-j \varepsilon \gamma_{12} \bar{\Omega}^{\alpha}}{\Gamma(1-\alpha)} \lim _{T \rightarrow \infty} \frac{1}{T} \int_{0}^{T}\left[\cos (i \bar{\tau}) \sin (j \bar{\tau}) \int_{0}^{\bar{\tau}} \frac{\cos (j s)}{s^{\alpha}} d s\right] d \bar{\tau} \\
& -\frac{-j \varepsilon \gamma_{12} \bar{\Omega}^{\alpha}}{\Gamma(1-\alpha)} \lim _{T \rightarrow \infty} \frac{1}{T} \int_{0}^{T}\left[\cos (i \bar{\tau}) \cos (j \bar{\tau}) \int_{0}^{\bar{\tau}} \frac{\sin (j s)}{s^{\alpha}} d s\right] d \bar{\tau} .
\end{aligned}
$$

By defining the first part of Eq. (A11) as $U_{1}$ and the second part as $U_{2}$ and integrating them by parts one obtains

$$
\begin{aligned}
U_{1}= & \frac{-j \varepsilon \gamma_{12} \bar{\Omega}^{\alpha}}{\Gamma(1-\alpha)} \lim _{T \rightarrow \infty} \frac{1}{T} \int_{0}^{T}\left\{\frac{1}{2}[\sin (i+j) \bar{\tau}-\sin (i-j) \bar{\tau}] \int_{0}^{\bar{\tau}} \frac{\cos (j s)}{s^{\alpha}} d s\right\} d \bar{\tau} \\
= & \left.\frac{-j \varepsilon \gamma_{12} \bar{\Omega}^{\alpha}}{2 \Gamma(1-\alpha)} \lim _{T \rightarrow \infty} \frac{1}{T}\left[\frac{\cos (i+j) \bar{\tau}}{i+j}-\frac{\cos (i-j) \bar{\tau}}{i-j}\right] \int_{0}^{\bar{\tau}} \frac{\cos (j s)}{s^{\alpha}} d s\right|_{0} ^{T} \\
& -\frac{j \varepsilon \gamma_{12} \bar{\Omega}^{\alpha}}{2 \Gamma(1-\alpha)} \lim _{T \rightarrow \infty} \frac{1}{T} \int_{0}^{T}\left[\frac{\cos (i+j) \bar{\tau}}{i+j}-\frac{\cos (i-j) \bar{\tau}}{i-j}\right] \frac{\cos (j \bar{\tau})}{\bar{\tau}^{\alpha}} d \bar{\tau} \\
U_{2}= & \frac{-j \varepsilon \gamma_{12} \bar{\Omega}^{\alpha}}{\Gamma(1-\alpha)} \lim _{T \rightarrow \infty} \frac{1}{T} \int_{0}^{T}\left\{\frac{1}{2}[\cos (i+j) \bar{\tau}-\cos (i-j) \bar{\tau}] \int_{0}^{\bar{\tau}} \frac{\sin (j s)}{s^{\alpha}} d s\right\} d \bar{\tau} \\
= & \left.\frac{j \varepsilon \gamma_{12} \bar{\Omega}^{\alpha}}{2 \Gamma(1-\alpha)} \lim _{T \rightarrow \infty} \frac{1}{T}\left[\frac{\sin (i+j) \bar{\tau}}{i+j}+\frac{\sin (i-j) \bar{\tau}}{i-j}\right] \int_{0}^{\bar{\tau}} \frac{\sin (j s)}{s^{\alpha}} d s\right|_{0} ^{T} \\
& -\frac{j \varepsilon \gamma_{12} \bar{\Omega}^{\alpha}}{2 \Gamma(1-\alpha)} \lim _{T \rightarrow \infty} \frac{1}{T} \int_{0}^{T}\left[\frac{\sin (i+j) \bar{\tau}}{i+j}+\frac{\sin (i-j) \bar{\tau}}{i-j}\right] \frac{\sin (j \bar{\tau})}{\bar{\tau}^{\alpha}} d \bar{\tau}
\end{aligned}
$$

For the calculation procedure with the above equations, it should be noted that we adopted that $\frac{\sin (0 \bar{\tau})}{0}=1$ and $\frac{\cos (0 \bar{\tau})}{0}=0$. Further, we will introduce two important formulas that can be obtained from the residue theory, which are given as

$$
\begin{aligned}
& \lim _{T \rightarrow \infty} \int_{0}^{T} \frac{\sin (j p)}{p^{\alpha}} d p=j^{\alpha-1} \Gamma(1-\alpha) \cos \left(\frac{\alpha \pi}{2}\right) \\
& \lim _{T \rightarrow \infty} \int_{0}^{T} \frac{\cos (j p)}{p^{\alpha}} d p=j^{\alpha-1} \Gamma(1-\alpha) \sin \left(\frac{\alpha \pi}{2}\right)
\end{aligned}
$$

If we consider the above formula Eq. (A16) into the first part of Eq. (A13) denoted as $A_{U 11}$ and integrate the second part denoted as $A_{U 12}$, we obtain these terms in the following forms

$$
\begin{gathered}
A_{U 11}=\frac{\varepsilon \gamma_{12} j^{\alpha} \bar{\Omega}^{\alpha} \sin \left(\frac{\alpha \pi}{2}\right)}{2} \lim _{T \rightarrow \infty} \frac{1}{T}\left[\frac{\cos (i+j) T}{i+j}-\frac{\cos (i-j) T}{i-j}\right]=0, \\
A_{U 12}=-\frac{j \varepsilon \gamma_{12} \bar{\Omega}^{\alpha}}{2 \Gamma(1-\alpha)} \lim _{T \rightarrow \infty} \frac{1}{T} \int_{0}^{T}\left[\frac{\cos (i+j) \bar{\tau} \cos (j \bar{\tau})}{(i+j) \bar{\tau}^{\alpha}}-\frac{\cos (i-j) \bar{\tau} \cos (j \bar{\tau})}{(i-j) \bar{\tau}^{\alpha}}\right] d \bar{\tau}=0,
\end{gathered}
$$

Similarly, substituting Eq. (A15) into the first part of Eq. (A14) denoted as $A_{U 21}$ and integrating the second part denoted as $A_{U 22}$, we obtain these terms in the following forms

$$
\begin{array}{r}
A_{U 21}=\frac{\varepsilon \gamma_{12} j^{\alpha} \bar{\Omega}^{\alpha} \cos \left(\frac{\alpha \pi}{2}\right)}{2} \lim _{T \rightarrow \infty} \frac{1}{T}\left[\frac{\sin (i+j) T}{i+j}+\frac{\sin (i-j) T}{i-j}\right] \\
= \begin{cases}0, & i \neq j \\
\frac{\varepsilon \gamma_{12} j^{\alpha} \bar{\Omega}^{\alpha} \cos \left(\frac{\alpha \pi}{2}\right)}{2}, & i=j\end{cases}
\end{array}
$$




$$
A_{U 22}=-\frac{j \varepsilon \gamma_{12} \bar{\Omega}^{\alpha}}{2 \Gamma(1-\alpha)} \lim _{T \rightarrow \infty} \frac{1}{T} \int_{0}^{T}\left[\frac{\sin (i+j) \bar{\tau} \sin (j \bar{\tau})}{(i+j) \bar{\tau}^{\alpha}}+\frac{\sin (i-j) \bar{\tau} \sin (j \bar{\tau})}{(i-j) \bar{\tau}^{\alpha}}\right] d \bar{\tau}=0,
$$

By combining Eqs. (A12)-(A14) and Eqs. (A17)-(A20) one obtains

$$
\left[M_{11}\right]_{i j}^{\alpha}= \begin{cases}0, & i \neq j \\ \frac{\varepsilon \gamma_{12} j^{\alpha} \bar{\Omega}^{\alpha} \cos \left(\frac{\alpha \pi}{2}\right)}{2}, & i=j\end{cases}
$$

Other elements of the matrix $\boldsymbol{M}_{2}^{\alpha}$ and vectors $\boldsymbol{R}_{2}^{\alpha}$ and $\boldsymbol{V}_{2}^{\alpha}$ can be obtained using a similar procedure as given in Eqs. (A11)-(A20). The final values of these elements are given in Eqs. (72). 